J. Math. Biol. manuscript No.

(will be inserted by the editor)

\title{
Signal propagation in the MAPK cascade: role of bistability and ultrasensitivity for a mixed problem
}

\author{
Ralf Blossey · Anne Devys · Thierry Goudon · Pauline Lafitte
}

Received: date / Accepted: date

\begin{abstract}
The MAPK signaling cascade is nowadays understood as a network module highly conserved across species. Its main function is to transfer a signal arriving at the plasma membrane to the cellular interior, the nucleus. Current understanding of 'how' this is achieved involve the notions of ultrasensitivity and bistability which relate to the nonlinear dynamics of the biochemical network, ignoring spatial aspects. Much less, indeed, is so far known about the propagation of the signal through the cytoplasm. In this work we formulate, starting from a Michaelis-Menten model for the MAPK cascade in Xenopus oocytes, a reaction-diffusion model of the cascade. We study this model in one space dimension. Basing ourselves on previous general results on reaction diffusion models, we particularly study for our model the conditions for signal propagation. We show that the existence of a propagating front depends sensitively on the initial and boundary conditions at the plasma membrane. Possible biological consequences of this finding are discussed.
\end{abstract}

Keywords Cell signalling $\cdot$ MAPK cascade $\cdot$ Reaction-Diffusion equations $\cdot$ Wave front

\section{Introduction and formulation of the problem}

In general, a signal (caused e.g. by a hormone) arriving at the plasma membrane of the cell needs to be transferred to the cell nucleus in order to excite a coherent cellular response: see the illustration in Figure 1. Dedicated signalling chains have evolved to achieve this task. In what follows we are particularly interested in a prominent one, which is the MAP Kinase signalling chain. The acronym MAP stands for mitogen-activated protein, whereby a mitogen is a factor that stimulates mitosis. This signalling chain is stimulated by the provision of a hormone at a membrane receptor, which in turn activates the protein Ras. Downstream of Ras is

Ralf Blossey

Institut de Recherche Interdisciplinaire, CNRS USR3078, Parc de la Haute Borne, 50 avenue de Halley, BP 70478, F-59658 Villeneuve d'Ascq Cedex, France

Tel.:+33 362531731

Fax:+33 362531790

E-mail: ralf.blossey@iri.univ-lille1.fr

Anne Devys · Thierry Goudon · Pauline Lafitte

INRIA Lille Nord Europe Research Centre, Parc de la Haute Borne, 40, avenue Halley, F-59650 Villeneuve d'Ascq cedex, France \& Laboratoire Paul Painleve, UMR 8524, USTL - Cite Scientifique, F-59655 Villeneuve d'Ascq cedex, France

Tel.: +33 359577889

Fax: +33320210776

E-mail: anne.devys@math.univ-lille1.fr, thierry.goudon@inria.fr, pauline.lafitte@math.univ-lille1.fr 
a cascade of further protein phosphorylations which ultimately interacts with the transcription machinery in the cell nucleus. One class of downstream kinases is called MAP Kinase (MAPK). Upstream of MAP kinases are another family of proteins, MAP2K (MAP Kinase Kinase), and still upstream are the MAP3K proteins (MAP Kinase Kinase Kinase), see Figure 1. Thus we understand the hormonal response as a cascade of protein phosphorylations whose typical like behaviour is that only a signal above a concentration threshold is significanty amplified in order to obtain a sustained cellular reponse. In addition, the cascade is commonly controlled by feedback loops which allow it to perform in a bistable, i.e. 'all-or-none' like fashion, see [7].

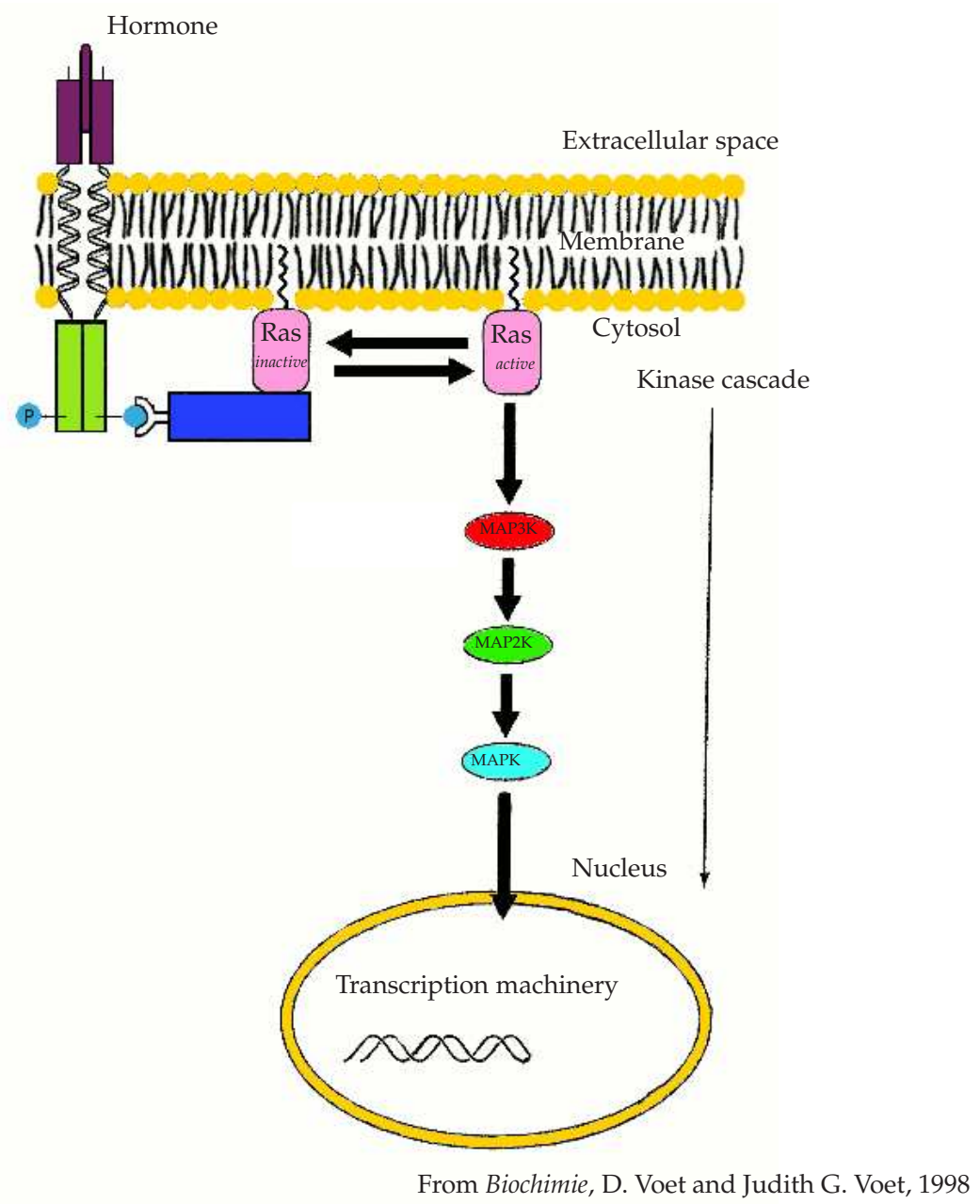

Fig. 1 A schematic view of the MAPK cascade.

While a whole body of work exists on the biochemical kinetics of the cascade, very little is so far known about how the signal traverses the intracellular space. It is commonly assumed that both diffusion in the cytoplasm as well as dedicated machineries like molecular motors are involved in signal transport. Clearly, the level of complexity of these mechanisms depends on the level of evolution of the cell, e.g. whether the cell is 
a prokaryote (e.g., a bacterium), or an eukaryote (a nuclear cell), and what is the corresponding differentiation status and functionality in the latter case.

Recently, a number of papers have appeared which explicitly address aspects of the spatial propagation of the MAPK cascade, see $[6,4,9]$ and the references therein. In this work we study a simple model for signal transport in a cytoplasm, motivated by the MAPK cascade. The model is a straightforward extension of a kinetic model proposed by Angeli et al. [1] for the MAPK cascade. A characteristic feature of this model is the bistability of the cascade, and it is particularly this feature that we exploit in our model.

The model we develop is based on the usual reaction kinetics of the MAPK cascade in which an upstream kinase activates a downstream one at two residues. For this kinetics, either a mass-action law or a MichaelisMenten kinetics can be assumed. The model is described in more details in Appendix A. In this way, one obtains the usual system of ordinary differential equations for the concentrations of MAPK and MAP2K, together with conservation laws for their total concentrations. In order to arrive at a spatial model, we require the following (simplifying) assumptions.

Assumption 1. We assume that molecular transport in the cytoplasm does not involve the action of molecular motors or other specialised molecular structures in the cell.

As a result of this assumption, the main mechanism of transport is molecular diffusion.

Assumption 2. We assume that chemical reactions (phosphorylations) in the cascade are always to be considered as 'fast' in the sense that at every instant in time, the cascade molecules are in chemical equilibrium.

As a consequence of Assumption 2, the concentrations of all molecular species can be expressed as simple functions of the molecule at cascade entry which initializes the signaling chain. Neither MAP2K nor MAPK are therefore considered as diffusing quantities.

Based on these two assumptions, we can immediately propose a model for the transport. Let $m(x, t)$ be the concentration of the molecule at cascade entry, the MAP3K in Figure 1. For the MAPK cascade in Xenopus oocytes, this is the molecule Mos. We then have

$$
\partial_{t} m(x, t)-D \Delta m(x, t)=R[m(x, t)]
$$

in which the diffusion operator on the left is balanced by a reaction-term $R[m(x, t)]$. The form of $R[m(x, t)]$ can be computed exactly for a simple model of the MAPK cascade, both for the case of a linear kinetics and a Michaelis-Menten kinetics (see Appendix A). For our present purposes, it suffices to sketch a qualitative shape, which can be inferred from Angeli's paper (see Figure 2). Here, $R[m(x, t)]$ is written as the sum of two terms, one of which has a sigmoidal shape, the other of which is a linear degradation term. Thus we have

$$
R[m(x, t)]=\Sigma[m(x, t)]-\gamma m(x, t)
$$

with

$$
\Sigma[m]=\frac{a \mathfrak{M} m^{4}}{\mathfrak{M}^{4}+\mathfrak{M}^{2} m^{2}+m^{4}},
$$

where $a$ is the inverse of the characteristic time of creation of Mos, $\gamma$ is the inverse of the characteristic time of its degradation and $\mathfrak{M}$ is the typical concentration. As it will be described in Appendix $\mathrm{A}$, the expression of the reaction term $\Sigma[\mathrm{m}]$ accounts for the feedback loop that drives the concentration of Mos depending on the MAPK concentration.

We call the Hill exponent $h$ the degree of the numerator and of the denominator (which are equal to obtain the sigmoidal shape) of the non-linearity (1.3). It is linked to the number of proteins involved in the cascade. 
The notion of ultrasensitivity refers to the steepness of the sigmoidal response curve which is characteristic of a signaling cascade: A very non linear response ensures an effective response to the signal received by the cell. The value $h=4$ is typical for the MAPK cascade, but in principle of course other values $h>1$ are equally possible. The limiting case $h=1$ corresponds to a degenerate case, a non-cooperative cascade which is not of biological interest.

We are interested in the following questions:

i) Under what conditions does the system have three stationary states (two stable, one unstable)?

ii) Under what conditions does the system have propagating states, i.e., the build-up of a profile connecting the two stable states and propagating from the cell surface to the nucleus?

iii) How does the propagation of the signal depend on the form of the non-linearity, and how does it depend on the chosen initial/boundary condition?

iv) What is the selected propagation speed?

As we shall see in the next Section, by rendering the problem (1.1)-(1.3) dimensionless we extract a single significant parameter, denoted by $\alpha$, which governs the dynamics. In Section 3 , we will prove that there exists a constant $\alpha_{c}$ such that for any $\alpha>\alpha_{c}$ there exist three stationary states : $0<a_{\alpha}<b_{\alpha}$. The dependence of $a_{\alpha}$ and $b_{\alpha}$ on $\alpha$ is also explored. The stability of these stationary states is studied:

a) $a_{\alpha}$ is unstable (Lemma 3 ) and 0 is locally stable (Theorem 2);

b) for $b_{\alpha}$ no such precise result is obtained: we can exhibit sufficient conditions for stability on the initial condition (Theorem 3) and on the boundary condition (Theorem 4). In short, one has to inject sufficient mass into the system in order to reach the stationary state $b_{\alpha}$. However, one has to point out that these conditions are not explicit enough to be numerically tractable.

Next, we investigate the existence of profiles connecting the stationary states: in Section 4, we prove the existence of a trajectory $T_{c^{*}}$ connecting the two stationary states 0 and $b_{\alpha}$, linked to an asymptotic speed of propagation $c^{*}$ which depends only on the non-linearity. It is independent of either the initial condition or the boundary condition. Note that in our case, we do not have an explicit formula for the asymptotic velocity as a function of the parameters. Theorem 6 shows the existence of a wave front with velocity $c^{*}$ connecting the two stationary states 0 and $b_{\alpha}$ provided that the conditions evoked in $\mathrm{b}$ ) are satisfied. Eventually, we end with numerical simulations in Section 5.1 exploring the behavior of the solution for several boundary and initial conditions. In Section 5.2 we compute numerically the asymptotic velocity $c^{*}$ and describe its dependence on the parameter $\alpha$.

\section{Dimensionless equations}

In order to complete the mathematical analysis of the problem, let us write the problem (1.1)-(1.3) in a dimensionless way. Note that the constants $a$ and $\gamma$ have the same dimension, that is, one over a time. In the same way, $\mathfrak{M}$ has the same dimension as $m$. As usual, the diffusion constant $D$ is homogeneous to the square of a length divided by a time. We set now:

$$
\bar{t}=\gamma t, \quad \bar{m}=\frac{m}{\mathfrak{M}^{\prime}}, \quad \bar{x}=\sqrt{\frac{\gamma}{D}} x, \quad \alpha=\frac{a}{\gamma} .
$$

Omitting the overlines the system takes the following dimensionless form:

$$
\partial_{t} m-\Delta m=z_{\alpha}(m)-m,
$$


where

$$
z_{\alpha}(m)=\frac{\alpha m^{4}}{m^{4}+m^{2}+1} .
$$

Note that in this dimensionless form all the information is contained in the parameter $\alpha$. Thus from now, the discussion will mainly concern this parameter $\alpha$. The problem (2.1) is set on $t \geq 0$ and $x \in[0, L]$. Biologically, $L$ represents the distance between the cell membrane and the nuclear membrane. Hence the problem has to be completed by an initial condition:

$$
m(0, x)=m_{0}(x) \quad \text { on } \quad[0, L]
$$

and boundary conditions:

- At $x=0$, we have

$$
m(t, 0)=\psi(t)
$$

- At $x=L<\infty$ we choose a free outgoing condition so that we impose $\partial_{x} m(x=L)=0$ (Neumann condition). However for the convenience of mathematical analysis we simply set $L=+\infty$.

\section{Stability analysis}

From now on, we note

$$
f_{\alpha}(m)=z_{\alpha}(m)-m
$$

\subsection{Preliminary remarks}

A plot of the two functions $m \mapsto z_{\alpha}(m)$ and $m \mapsto m$ reveals, under conditions to be detailed below, three intersections. These correspond to stationary states of the kinetics for homogeneous profiles $m$. Two of the states, for small and large values of $m$, are stable, while the intermediate state is unstable. The profile of $R[m(x, t)]$ thus corresponds to the reaction term of a bistable system.

If we plot the curve corresponding to the function $z: m \mapsto z_{\alpha}(m)$ and the line $y=m$, see Figure 2 we note that these two curves can have one or three distinct intersection points depending on the value of $\alpha$.

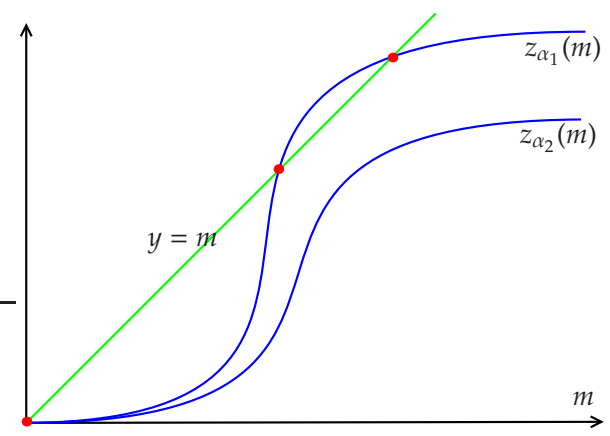

Fig. 2 Functions $z_{\alpha_{1}}$ and $z_{\alpha_{1}}$ with $\alpha_{1}>\alpha_{2}$ and the line $y=m$.

Let us now evaluate $\alpha_{0}$ such that if $\alpha>\alpha_{0}$ then there exist three stationary points. Let us denote

$$
f_{\alpha}(m)=\frac{m}{1+m^{2}+m^{4}} \underbrace{\left(\alpha m^{3}-\left(1+m^{2}+m^{4}\right)\right)}_{P_{\alpha}(m)},
$$


thus

$$
P_{\alpha}^{\prime}(m)=3 \alpha m^{2}-\left(2 m+4 m^{3}\right)=m\left(-4 m^{2}+3 \alpha m-2\right)
$$

It is easy to see that if $f$ has three non negative roots, then necessarily $\Delta=9 \alpha^{2}-32>0$. Thus, denoting $0<m_{1}<m_{2}$ the three non negative roots of $P_{\alpha}^{\prime}(m)$, a sufficient condition to have three stationary points is $f_{\alpha}\left(m_{2}\right)>0$. Numerically we find:

$$
\alpha_{0}=2.463 \text {. }
$$

In case of $\alpha>\alpha_{0}, f_{\alpha}$ vanishes at $0, a_{\alpha}$ and $b_{\alpha}$. We plot the shape of the graph of $f_{\alpha}$, in Figure 3 .

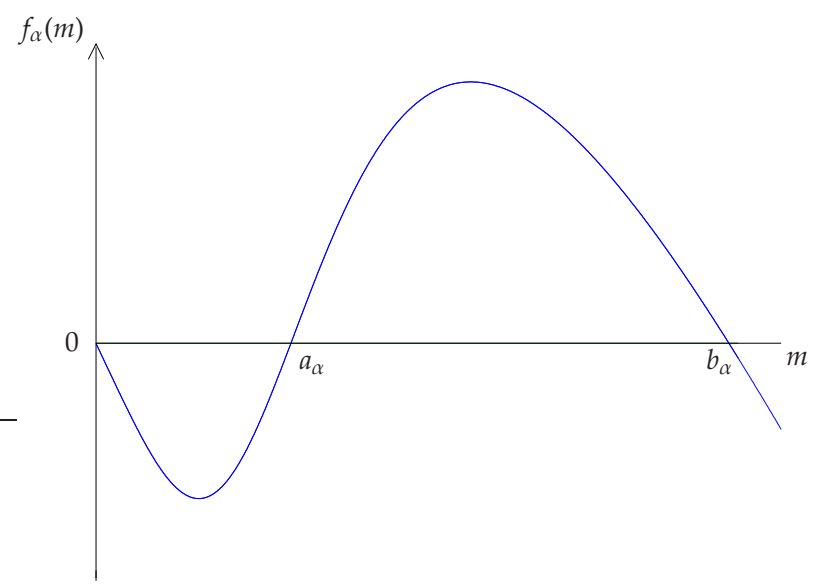

Fig. 3 Function $f_{\alpha}$.

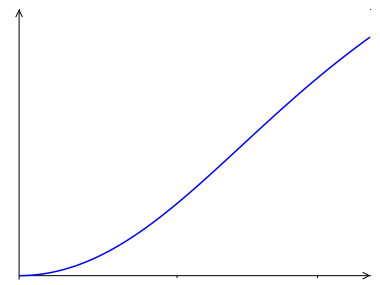

(a) $\alpha=2<\alpha_{0}$

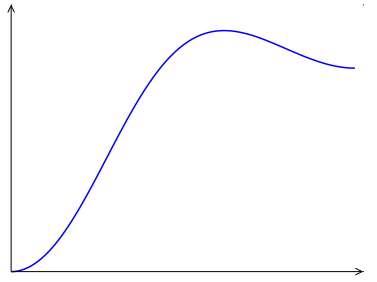

(b) $\alpha_{0}<\alpha=2.6<\alpha_{0}$

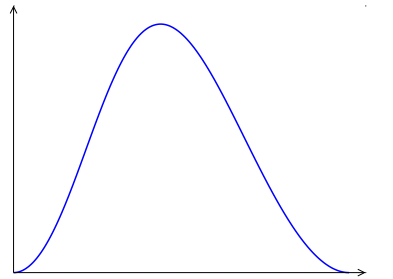

(c) $\alpha=\alpha_{c}$

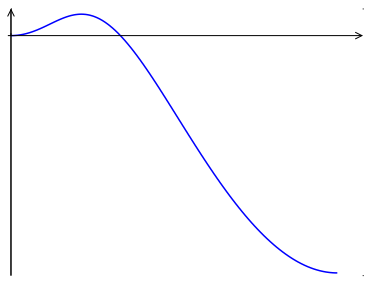

(d) $\alpha=4>\alpha_{c}$

Fig. 4 Function $-F_{\alpha}$ for three different values of $\alpha$

We set

$$
F_{\alpha}(x):=\int_{0}^{x} f_{\alpha}(u) \mathrm{d} u=\alpha x-\frac{x^{2}}{2}-\alpha \frac{1}{\sqrt{3}}\left(\arctan \left(\frac{2}{\sqrt{3}}\left(x+\frac{1}{2}\right)\right)+\arctan \left(\frac{2}{\sqrt{3}}\left(x-\frac{1}{2}\right)\right)\right) .
$$

The quantity $-F_{\alpha}$ is naturally interpreted as a mechanical potential of the system. Figure 4 represents the shape of the potential in the three different cases $\alpha<\alpha_{c}, \alpha=\alpha_{c}, \alpha>\alpha_{c}$ and makes the stable states appear, 


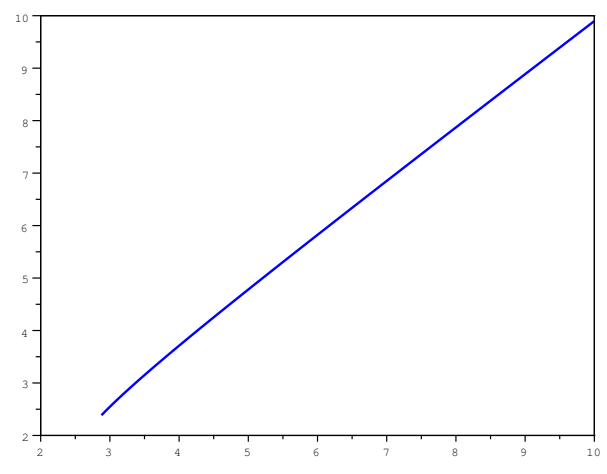

(a) $\alpha \mapsto b_{\alpha}$

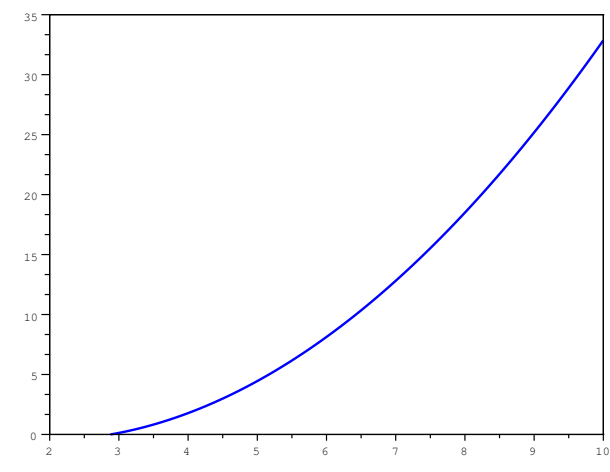

(b) $\alpha \mapsto F_{\alpha}\left(b_{\alpha}\right)$

Fig. $5 \alpha \mapsto b_{\alpha}$ and $\alpha \mapsto F_{\alpha}\left(b_{\alpha}\right)$ for $\alpha_{c}<\alpha<10$

where $\alpha_{c}>\alpha_{0}$ satisfies : $F_{\alpha_{c}}=\int_{0}^{b_{\alpha_{c}}} f_{\alpha_{c}}(u) \mathrm{d} u=0$. Numerically we find :

$$
\alpha_{c}=2.878
$$

Furthermore, we impose

$$
\int_{0}^{b_{\alpha}} f_{\alpha}(u) \mathrm{d} u>0
$$

Referring to Figure 4 , this condition means that the stable state $b_{\alpha}$ has negative and minimal energy $\left(-F_{\alpha}\left(b_{\alpha}\right)<\right.$ $0)$. Thanks to this condition we have:

$$
\exists \kappa_{\alpha} \in\left(a_{\alpha}, b_{\alpha}\right) \text { s.t. } F_{\alpha}\left(\kappa_{\alpha}\right)=\int_{0}^{\kappa_{\alpha}} f_{\alpha}(u) \mathrm{d} u=0,
$$

and then $\forall q \in\left(\kappa_{\alpha}, b_{\alpha}\right), F_{\alpha}(q)>0$ and $F_{\alpha}^{\prime}(q)=f_{\alpha}(q)>0$.

Thanks to Ferrari's formula we can give an explicit equivalent of the biggest root of $f_{\alpha}$ that we denote $b_{\alpha}$. After a simple computation we find out that

$$
b_{\alpha} \sim \alpha
$$

and thus we get:

$$
\kappa_{\alpha} \sim \frac{\alpha^{2}}{2}
$$

To illustrate we plot $\alpha \mapsto b_{\alpha}$ and $\alpha \mapsto F_{\alpha}\left(B_{\alpha}\right)$ in Figure 5. From this point on, we will omit the index $\alpha$.

3.2 Study of the initial-boundary value problem (2.1)-(2.3) in the quarter-space $\mathbb{R}^{+} \times \mathbb{R}^{+}$

The problem (2.1)-(2.3) is rewritten just below:

$$
\left\{\begin{array}{l}
m_{t}=\Delta m+f(m) \text { in } \mathbb{R}^{+} \times \mathbb{R}^{+} \\
m(x, 0)=m_{0}(x) \text { in } \mathbb{R}^{+} \\
m(0, t)=\psi(t) \quad \text { in } \mathbb{R}^{+}
\end{array}\right.
$$


where $\psi$ is a given function, $0 \leq \psi \leq b$. To be consistent we suppose

$$
\psi(0)=m_{0}(0)
$$

\subsubsection{A maximum principle.}

Two lemmas are very useful to the study of (3.8). They are given here without any proof, for more details see [2]. Lemma 1 is a version of the maximum principle.

Lemma 1 Let $u: \mathbb{R} \times[0, T] \longrightarrow[0, b]$ and $v: \mathbb{R} \times[0, T] \longrightarrow[0, b]$ satisfy the inequalities

$$
\begin{array}{cl}
u_{t}-\Delta u-f(u) \geq v_{t}-\Delta v-f(v) & \text { in }\left(x_{1}, x_{2}\right) \times(0, T] \\
0 \leq v(x, 0) \leq u(x, 0) \leq b & \text { in }\left(x_{1}, x_{2}\right)
\end{array}
$$

where $-\infty \leq x_{1}<x_{2} \leq+\infty$ and $0 \leq T \leq \infty$. Moreover, if $x_{1}>-\infty$, assume that

$$
0 \leq v\left(x_{1}, t\right) \leq u\left(x_{1}, t\right) \leq b \text { on }[0, T]
$$

and if $x_{2}<\infty$ assume that

$$
0 \leq v\left(x_{2}, t\right) \leq u\left(x_{2}, t\right) \leq b \text { on }[0, T] .
$$

Then $u \geq v$, and if $u(x, 0)>v(x, 0)$ in an open sub-interval of $\left(x_{1}, x_{2}\right)$ then $u>v$, in $\left(x_{1}, x_{2}\right) \times(0, T]$.

Lemma 2 Let $q: \mathbb{R} \longrightarrow[0, b]$ be a solution of the equation $q^{\prime \prime}+f(q)=0$ in $\left(x_{1}, x_{2}\right)$ with $x_{1}>0$, and let $q\left(x_{1}\right)=q\left(x_{2}\right)=0$. Let $v(x, t)$ denote the solution of the initial-boundary value problem

$$
\begin{gathered}
v_{t}=\Delta v+f(v) \text { in } \mathbb{R}^{+} \times \mathbb{R}^{+} \\
v(x, 0)= \begin{cases}q(x) & \text { in }\left(x_{1}, x_{2}\right) \\
0 & \text { in } \mathbb{R}^{+} \backslash\left(x_{1}, x_{2}\right)\end{cases} \\
v(0, t)=\phi(t) \text { in } \mathbb{R}^{+},
\end{gathered}
$$

where $\phi$ is supposed nondecreasing and such that $\phi(0)=0$ and $0 \leq \phi \leq b$. Then $v$ is nondecreasing with respect to tand

$$
\lim _{t \rightarrow \infty} v(x, t)=\tau(x)
$$

where $\tau$ is the smallest nonnegative solution of

$$
\tau^{\prime \prime}+f(\tau)=0 \text { in } \mathbb{R}^{+}
$$

which satisfies the inequalities

$$
\tau(0) \geq \lim _{t \rightarrow \infty} \phi(t) \quad \text { and } \quad \tau(x) \geq q(x) \text { in }\left(x_{1}, x_{2}\right) .
$$

Moreover, the convergence of $v$ to $\tau$ is uniform on each closed bounded interval in the interior of $\mathbb{R}^{+}$. 


\subsubsection{Stability and threshold results}

In our case, since $f^{\prime}(0)<0$, we will see that we need a minimal amount of mass injected in the system through the boundary to reach the stable stationary point $x=b$. In this section, we give two results : the first one shows that if the boundary condition is too small in $L^{\infty}$-norm then the solution of (3.8) tends uniformly to zero as $t$ tends to infinity. The second shows that if the boundary condition combines a long enough support and a not too small value on this support then we observe a wave front.

For $v \in[0, \kappa)$ (where $\kappa$ is defined at (3.5)), let us denote $q_{v}$ a solution of

$$
q_{v}^{\prime \prime}+f\left(q_{v}\right)=0 \quad \text { in } \mathbb{R}^{+}
$$

with $q_{v}(0)=v$. In our case there is a solution of (3.9) such that

$$
\lim _{x \rightarrow \infty} q_{v}(x)=0
$$

In fact, choosing $q_{v}^{\prime}(0)=-\sqrt{-2 F(v)}$, where $F$ is the primitive of $f$ which vanishes at 0 (see (3.2)), one can prove using the first integral

$$
\frac{1}{2} q_{v}^{\prime 2}+F\left(q_{v}\right)=0
$$

that $q_{v}$ converges to zero.

Theorem 1 Let $m: \mathbb{R}^{+} \times \mathbb{R}^{+} \longrightarrow[0, b]$ be the solution of the problem (3.8) with $m_{0} \equiv 0$. If

$$
v=\sup _{t \in \mathbb{R}^{+}} \psi(t)<\kappa
$$

then $m(x, t) \leq q_{v}(x)$. In particular,

$$
\lim _{x \rightarrow \infty} \limsup _{t \rightarrow \infty} m(x, t)=0
$$

Proof It is a consequence of Lemma 1.

The following Lemma shows that the stationary states $a$ is unstable and 0 is locally stable.

Lemma 3 Let $m: \mathbb{R}^{+} \times \mathbb{R}^{+} \longrightarrow[0, b]$ be a solution of $(3.8)$ in $\mathbb{R}^{+} \times \mathbb{R}^{+}$and $f(u)<0$ in $(0, \gamma]$. If $m_{0}: \mathbb{R}^{+} \longrightarrow[0, \gamma]$ and $\sup _{\mathbb{R}^{+}} \psi(t) \leq \gamma$, then

$$
\lim _{x \rightarrow \infty} \limsup _{t \rightarrow \infty} m(x, t)=0
$$

Proof Step1: Let $w$ be solution of

$$
\begin{aligned}
w_{t} & =\Delta w+f(w) & & \text { in } \mathbb{R}^{+} \times \mathbb{R}^{+}, \\
w(x, 0) & =\gamma & & \text { in } \mathbb{R}^{+}, \\
w(0, t) & =\gamma & & \text { in } \mathbb{R}^{+} .
\end{aligned}
$$

Applying Lemma 1 we get $m(x, t) \leq w(x, t)$ in $\mathbb{R}^{+} \times \mathbb{R}^{+}$.

Step 2: Since $f(0)=f(a)=0$, Lemma 1 shows that $0 \leq w(x, t) \leq a$ in $\mathbb{R}^{+} \times \mathbb{R}^{+}$. Furthermore note that $f \leq 0$ on $\overline{[0, a] \text { thus }}$

$$
w_{t}-\Delta w \leq w_{t}-\Delta w-f(w)=0=u_{t}-\Delta u,
$$

where $u \equiv \gamma$ in $\mathbb{R}^{+} \times \mathbb{R}^{+}$. Applying again Lemma 1 , we get $w(x, t) \leq \gamma$ in $\mathbb{R}^{+} \times \mathbb{R}^{+}$. 


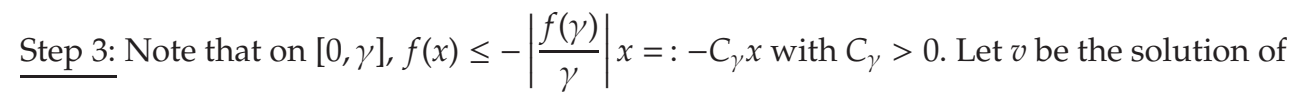

$$
\begin{aligned}
v_{t} & =\Delta v-C_{\gamma} v & & \text { in } \mathbb{R}^{+} \times \mathbb{R}^{+}, \\
v(x, 0) & =\gamma & & \text { in } \mathbb{R}^{+}, \\
v(0, t) & =\gamma & & \text { in } \mathbb{R}^{+} .
\end{aligned}
$$

Using again Lemma 1 , we show that $w(x, t) \leq v(x, t)$ thus $m(x, t) \leq v(x, t)$ in $\mathbb{R}^{+} \times \mathbb{R}^{+}$.

Step 4: Finally, $\bar{v}(x, t)=e^{C_{\gamma} t}\left(v(x, t)-\gamma e^{-\sqrt{C_{\gamma}} x}\right)$ is the solution of

$$
\begin{aligned}
\bar{v}_{t} & =\Delta \bar{v} & & \text { in } \mathbb{R}^{+} \times \mathbb{R}^{+}, \\
\bar{v}(x, 0) & =\gamma\left(1-e^{-\sqrt{C_{\gamma}} x}\right) & & \text { in } \mathbb{R}^{+}, \\
\bar{v}(0, t) & =0 & & \text { in } \mathbb{R}^{+} .
\end{aligned}
$$

It is easy to see that $\bar{v}(x, t) \leq \gamma$ in $\mathbb{R}^{+} \times \mathbb{R}^{+}$, thus $\lim _{t \rightarrow \infty} v(x, t)-\gamma e^{-\sqrt{C_{\gamma}} x}=0$ and we conclude that

$$
\lim _{x \rightarrow \infty} \limsup _{t \rightarrow \infty} m(x, t)=0
$$

For any $\rho \in[0, a)$ we define

$$
s(\rho)=\sup \left\{\frac{f(u)}{u-\rho}, u \in(a, b)\right\}
$$

Notation 1 In what follows we shall use the following usual notation:

$$
[u]^{+}=\max (u, 0)
$$

Theorem 2 Let $m(x, t): \mathbb{R}^{+} \times \mathbb{R}^{+} \mapsto[0, b]$ be the solution of (3.8). Suppose that for some $T \in \mathbb{R}^{+}$and some $\rho \in(0, a)$,

$$
\psi(t) \leq \rho \quad \text { in } \quad(T, \infty)
$$

and

$$
e^{s(\rho) T}\left(\sqrt{\frac{e s(\rho)}{4 \pi(s(\rho) T+1)}} \int_{0}^{\infty}\left[m_{0}(y)-\rho\right]^{+} \mathrm{d} y+s(\rho) \sqrt{\frac{e}{2 \pi}} \int_{0}^{T} e^{-s(\rho) t}[\psi(t)-\rho]^{+} \mathrm{d} t\right)<a-\rho
$$

Then

$$
\lim _{x \rightarrow \infty} \limsup _{t \rightarrow \infty} m(x, t)=0
$$

Proof Fix $\rho \in[0, a)$.

Step 1 : Construction of an upper-solution $w$. Let $w(x, t)$ be the solution of

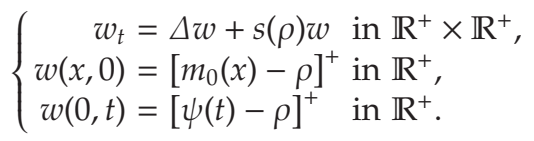

Using Lemma 1 we get $w \geq 0$ and then $w=[w]^{+}$. Since $s(\rho)>0$ and $f(u)<0$ in $(0, a)$ we have $f(m) \leq s(\rho)[m-\rho]^{+}$. Let $v(x, t):=m(x, t)-\rho$, then

$$
v_{t}-\Delta v-s(\rho)[v]^{+} \leq m_{t}-\Delta m-f(m)=0=w_{t}-\Delta w-s(\rho)[w]^{+} .
$$


Using Lemma 1 again we get $v(x, t) \leq w(x, t)$.

Step 2 : There exists $\eta$ such that $w+\rho \leq \eta<a$. Let us define for $(x, t) \in \mathbb{R}^{+} \times \mathbb{R}^{+}$,

$$
\bar{w}(x, t):=w(x, t) e^{-s(\rho) t}
$$

and

Then $W$ satisfies

$$
W(x, t)=\bar{w}(x, t)-[\psi(t)-\rho]^{+} e^{-s(\rho) t} .
$$

$$
\left\{\begin{array}{rlrl}
W_{t}-\Delta W & =-\partial_{t}\left([\psi(t)-\rho]^{+} e^{-s(\rho) t}\right) & & \text { in } \mathbb{R}^{+} \times \mathbb{R}^{+}, \\
W(x, 0) & =\left[m_{0}(x)-\rho\right]^{+}-[\psi(0)-\rho]^{+} & \text {in } \mathbb{R}^{+} \\
W(0, t) & =0 & & \text { in } \mathbb{R}^{+} .
\end{array}\right.
$$

Let us now extend function $W$ to $\mathbb{R}$ as an odd function:

$$
\left\{\begin{aligned}
\bar{W}_{t}-\Delta \bar{W} & =-\partial_{t}\left([\psi(t)-\rho]^{+} e^{-s(\rho) t}\right) s g(x) & & \text { in } \mathbb{R}^{+} \times \mathbb{R} \\
\bar{W}(x, 0) & =\left(\left[m_{0}(s g(x) x)-\rho\right]^{+}-[\psi(0)-\rho]^{+}\right) s g(x) & & \text { in } \mathbb{R} \\
\bar{W}(0, t) & =0 & & \text { in } \mathbb{R}^{+}
\end{aligned}\right.
$$

where

$$
s g(x)=\left\{\begin{array}{r}
1 \text { if } x \geq 0 \\
-1 \text { if } x<0
\end{array}\right.
$$

Note that

$$
\forall x \in \mathbb{R}^{+}, \quad \bar{W}(x)=W(x) .
$$

Omitting the overlines and using the heat kernel, the solution reads as follows:

$W(x, t)=\int_{\mathbb{R}} \frac{e^{-\frac{|x-y|^{2}}{4 t}}}{\sqrt{4 \pi t}}\left(\left[m_{0}(s g(y) y)-\rho\right]^{+}-[\psi(0)-\rho]^{+}\right) s g(y) \mathrm{d} y-\int_{0}^{t} \int_{\mathbb{R}} \frac{e^{-\frac{|x-y|^{2}}{(t+-\sigma)}}}{\sqrt{4 \pi(t-\sigma)}} \partial_{t}\left([\psi(\sigma)-\rho]^{+} e^{-s(\rho) \sigma}\right) s g(y) \mathrm{d} y \mathrm{~d} \sigma$.

Let us denote $g(\sigma)=[\psi(\sigma)-\rho]^{+} e^{-s(\rho) \sigma}$, and after an integration by parts, we get:

$$
\begin{aligned}
W(x, t) & =\int_{\mathbb{R}} \frac{e^{-\frac{|x-y|^{2}}{4 t}}}{\sqrt{4 \pi t}}\left[m_{0}(s g(y) y)-\rho\right]^{+} s g(y) \mathrm{d} y+\int_{0}^{t} \int_{\mathbb{R}} \frac{e^{-\frac{x-y{ }^{2}}{4(t-\sigma)}}}{8 \sqrt{\pi}(t-\sigma)^{3 / 2}}\left(-\frac{|x-y|^{2}}{t-\sigma}+2\right) \operatorname{sg}(y) \mathrm{d} y g(\sigma) \mathrm{d} \sigma \\
& =I_{1}(t)+\int_{0}^{t} I_{2}(\sigma) g(\sigma) \mathrm{d} \sigma .
\end{aligned}
$$

Let us find an upperbound for $I_{1}$ :

$$
\begin{aligned}
I_{1}(t) & =\int_{\mathbb{R}} \frac{e^{-\frac{|x-y|^{2}}{4 t}}}{\sqrt{4 \pi t}}\left[m_{0}(s g(y) y)-\rho\right]^{+} s g(y) \mathrm{d} y \\
& =\int_{0}^{\infty} \frac{e^{-\frac{x-\left.y\right|^{2}}{4 t}}}{\sqrt{4 \pi t}}\left[m_{0}(y)-\rho\right]^{+} \mathrm{d} y-\int_{-\infty}^{0} \frac{e^{-\frac{|x-y|^{2}}{4 t}}}{\sqrt{4 \pi t}}\left[m_{0}(-y)-\rho\right]^{+} \mathrm{d} y \\
& \leq \int_{0}^{\infty} \frac{e^{-\frac{x x-y^{2}}{4 t}}}{\sqrt{4 \pi t}}\left[m_{0}(y)-\rho\right]^{+} \mathrm{d} y \\
& \leq \frac{1}{\sqrt{4 \pi t}} \int_{0}^{\infty}\left[m_{0}(y)-\rho\right]^{+} \mathrm{d} y .
\end{aligned}
$$


In particular,

$$
I_{1}\left(T+\frac{1}{s(\rho)}\right) \leq \sqrt{\frac{s(\rho)}{4 \pi(s(\rho) T+1)}} \int_{0}^{\infty}\left[m_{0}(y)-\rho\right]^{+} \mathrm{d} y .
$$

Let us compute the integral in space $I_{2}$ :

$$
\begin{aligned}
I_{2} & =\int_{\mathbb{R}} \frac{e^{-\frac{|x-y|^{2}}{4(t-\sigma)}}}{8 \sqrt{\pi}(t-\sigma)^{3 / 2}}\left(-\frac{|x-y|^{2}}{t-\sigma}+2\right) s g(y) \mathrm{d} y \\
& =\int_{\mathbb{R}^{+}} \frac{e^{-\frac{|x-y|^{2}}{4(t-\sigma)}}}{8 \sqrt{\pi}(t-\sigma)^{3 / 2}}\left(-\frac{|x-y|^{2}}{t-\sigma}+2\right) \operatorname{sg}(y) \mathrm{d} y-\int_{\mathbb{R}^{-}} \frac{e^{-\frac{|x-y|^{2}}{4(t-\sigma)}}}{8 \sqrt{\pi}(t-\sigma)^{3 / 2}}\left(-\frac{|x-y|^{2}}{t-\sigma}+2\right) s g(y) \mathrm{d} y \\
& =\int_{\frac{-x}{2 \sqrt{t-\sigma}}}^{\frac{x}{2 \sqrt{t-\sigma}}} \frac{1}{2 \sqrt{\pi} \sqrt{t-\sigma}} e^{-z^{2}}\left(4 z^{2}+2\right) \mathrm{d} z \\
& =\left[\frac{z e^{-z^{2}}}{\sqrt{\pi}(t-\sigma)}\right]_{\frac{-x}{2 \sqrt{t-\sigma}}}^{\frac{x}{2 \sqrt{t-\sigma}}} \\
& \leq \frac{e^{-1 / 2}}{\sqrt{2 \pi}(t-\sigma)} .
\end{aligned}
$$

Finally, thanks to (3.11) we get

$$
\int_{0}^{T+\frac{1}{s(\rho)}} I_{2}(\sigma) g(\sigma) \mathrm{d} \sigma \leq \frac{s(\rho)}{\sqrt{2 e \pi}} \int_{0}^{T} e^{-s(\rho) t}[\psi(t)-\rho]^{+} \mathrm{d} t
$$

To conclude,

$$
\left.W\left(x, T+\frac{1}{s(\rho)}\right) \leq \sqrt{\frac{s(\rho)}{4 \pi(s(\rho) T+1)}} \int_{0}^{\infty}\left[m_{0}(y)-\rho\right]^{+} \mathrm{d} y+\frac{s(\rho)}{\sqrt{2 e \pi}}\right) \int_{0}^{T} e^{-s(\rho) t}[\psi(t)-\rho]^{+} \mathrm{d} t,
$$

which, thanks to (3.12), leads to

$$
\exists \eta<a, \quad \text { s.t. } \quad w(x, t)<\eta-\rho \quad(x, t) \in \mathbb{R}^{+} \times \mathbb{R}^{+} .
$$

We conclude using Lemma 3.

For any $\beta \in(\kappa, b)$, where $\kappa$ is defined at (3.5), we define the length

$$
\ell_{\beta}=2 \int_{0}^{\beta} \frac{1}{\sqrt{2(F(\beta)-F(u))}} \mathrm{d} u \text {. }
$$

Finally, let $q_{\beta}$ be the solution of

$$
q_{\beta}^{\prime \prime}+f\left(q_{\beta}\right)=0 \text { in } \mathbb{R}^{+},
$$

with

$$
q_{\beta}(0)=0 \quad \text { and } \quad \frac{1}{2} q_{\beta}^{\prime 2}+F\left(q_{\beta}\right)=F(\beta)
$$


These conditions imply

$$
q_{\beta}>0, q_{\beta}(0)=q_{\beta}\left(\ell_{\beta}\right)=0 \text { and } q_{\beta}(x) \leq q_{\beta}\left(\frac{\ell_{\beta}}{2}\right)=\beta \text { on }\left(0, \ell_{\beta}\right)
$$

For more details see Appendix B.

Theorem 3 Let $m: \mathbb{R}^{+} \times \mathbb{R}^{+} \longrightarrow[0, b]$ be the solution of (3.8). For some $\beta \in(\kappa, b)$ and some $x_{0}>0$ assume

$$
m_{0}(x) \geq q_{\beta}\left(x-x_{0}\right) \quad \text { on } \quad\left(x_{0}, x_{0}+\ell_{\beta}\right)
$$

then

$$
\lim _{x \rightarrow \infty} \lim _{t \rightarrow \infty} m(x, t)=b .
$$

Proof Step 1: We apply Lemma 2 with $q(x)=q_{\beta}(x)$ :

let define

$$
\begin{gathered}
v_{t}=\Delta v+f(v), \\
v(x, 0)= \begin{cases}q_{\beta}\left(x-x_{0}\right) & \text { on }\left(x_{0}, x_{0}+\ell_{\beta}\right) \\
0 & \text { on } \mathbb{R} \backslash\left(x_{0}, x_{0}+\ell_{\beta}\right),\end{cases} \\
v(0, t)=0 .
\end{gathered}
$$

Note that Lemma 1 shows that $m(x, t) \geq v(x, t)$. Furthermore, according to Lemma $2, t \mapsto v(x, t)$ is a nondecreasing function and

$$
\lim _{t \rightarrow \infty} v(x, t)=\tau(x),
$$

uniformly on each bounded interval in the interior of $\mathbb{R}^{+}$, where $\tau$ is the smallest non-negative function of

$$
\tau^{\prime \prime}+f(\tau)=0
$$

on $\mathbb{R}$ which satisfies $\tau(x) \geq q_{\beta}\left(x-x_{0}\right)$ on $\left(x_{0},+x_{0}+\ell_{\beta}\right)$.

Step 2: We now prove that $\tau(x) \notin[\kappa, b), \forall x \in \mathbb{R}$. Let us assume the contrary and get a contradiction. Suppose that

$$
\exists x_{0} \quad \text { s.t. } \quad r=\tau\left(x_{0}\right) \in[\kappa, b)
$$

then

$$
\frac{1}{2} \tau^{\prime 2}+F(\tau)=k \geq F(r)
$$

since $\tau^{\prime 2} \geq 0$. Thus $\frac{1}{\sqrt{k-F(u)}}$ is integrable on $[0, r]$ and $\tau(x)$ is implicitly defined by the equation

$$
x=x_{0} \pm \int_{\tau}^{r} \frac{\mathrm{d} u}{\sqrt{2(k-F(u))}}
$$

where the sign is determined by the sign of $\tau^{\prime}\left(x_{0}\right)$. Hence $\tau(x)$ vanishes with $\tau^{\prime} \neq 0$ at a finite value of $x$. Then $\tau$ cannot be nonnegative and we get a contradiction.

Step 3: Note that

$$
\tau\left(x_{0}+\frac{\ell_{\beta}}{2}\right) \geq q_{\beta}\left(\frac{\ell_{\beta}}{2}\right)=\beta>\kappa,
$$

then by continuity and because $\tau$ is the minimal solution, we get

$$
\tau(x) \equiv b,
$$

which proves the theorem. 
Theorem 4 Let $m: \mathbb{R}^{+} \times \mathbb{R}^{+} \longrightarrow[0, b]$ be the solution of (3.8). Let $\kappa$ defined at (3.5). If for any $\beta \in(\kappa, b)$ there is a positive time $T_{\beta}$ such that if

$$
\psi(t) \geq \beta \quad \text { on } \quad\left(t_{0}, t_{0}+T_{\beta}\right)
$$

for some nonnegative $t_{0}$, then

$$
\lim _{x \rightarrow \infty} \liminf _{t \rightarrow \infty} m(x, t)=b .
$$

Proof Step 1: Let $\chi$ be a smooth function such that

$$
\chi(t)=\left\{\begin{array}{l}
0 \text { on }(-\infty, 0), \\
\beta \text { on }(1, \infty) .
\end{array}\right.
$$

Let $w$ denote the solution of

$$
\begin{array}{ll}
w_{t}=\Delta w+f(w) & \text { in } \mathbb{R} \times \mathbb{R}^{+}, \\
w(x, 0)=m_{0}(x) & \text { in } \mathbb{R}^{+}, \\
w(0, t)=\chi(t) & \text { in } \mathbb{R}^{+} .
\end{array}
$$

Using Lemma 2, we get

$$
\lim _{t \rightarrow \infty} w(x, t)=\tau(x),
$$

where $\tau$ is the smallest nonnegative solution of

$$
\left\{\begin{array}{l}
\tau^{\prime \prime}+f(\tau)=0 \\
\tau(0)=\beta \text { and } \tau(x) \geq m_{0}(x) \text { on } \mathbb{R} .
\end{array}\right.
$$

Step 2: Let us show that the problem (3.17) has only one solution since $\beta>\kappa$. The first integral gives us

$$
\frac{1}{2}\left(\tau^{\prime}\right)^{2}+F(\tau)=\frac{1}{2}\left(\tau^{\prime}(0)\right)^{2}+F(\beta)=k \geq F(\beta)>0 .
$$

First of all, $\tau$ cannot vanish at some point: if $\tau\left(x_{0}\right)=0$, then $\left(\tau^{\prime}\left(x_{0}\right)\right)^{2}>0$ which is in contradiction with $\tau$ being nonnegative. Assume next that it exists $x_{0}$ such that $\tau^{\prime}\left(x_{0}\right)=0$, then

$$
\forall x \in \mathbb{R}, \quad \frac{1}{2}\left(\tau^{\prime}(x)\right)^{2}=F\left(\tau\left(x_{0}\right)\right)-F(\tau(x)) .
$$

Furthermore $F\left(\tau\left(x_{0}\right)\right)>F(\beta)$ and $F$ is increasing for $x>\beta$. This shows that $\tau^{\prime} \leq 0$ on $\left[x_{0}-h, x_{0}+h\right]$. Finally, $\forall x \in \mathbb{R}, \tau^{\prime} \leq 0$. This implies that $\tau^{\prime}(x)=-\sqrt{k-F(\tau)} \leq-\sqrt{k-F(\beta)}$ and necessarily, $\tau$ vanishes at some point which is a contradiction. Finally $\tau^{\prime}>0$ and then $\tau$ is increasing.

$\tau$ is increasing and bounded by $b$, it has a limit which is necessarily $b$ and according to the first integral we get

$$
\tau^{\prime}(0)=\sqrt{2(F(b)-F(\beta))}
$$

$\tau$ is then uniquely determined and since $\tau^{\prime}>0$ we have $\tau(x)>\beta$ on $\mathbb{R}_{*}^{+}$.

Step 3: Recall the function $q_{\beta}$ defined by (3.14). Since the convergence of $w(x, t)$ towards $\tau(x)$ as $t$ tends to $\overline{\text { infinity }}$ is uniform on each bounded interval, in particular, $w(x, t)$ converges to $\tau(x)$ uniformly on $\left[1,1+\ell_{\beta}\right]$, where $\ell_{\beta}$ is defined at (3.13). Thus there exists a time $T_{\beta}$ such that

$$
w\left(x, T_{\beta}\right) \geq q_{\beta}(x-1) \quad \text { on } \quad\left[1, \ell_{\beta}+1\right] .
$$

Futhermore, thanks to (3.16) $\psi\left(t+t_{0}\right) \geq \chi(t)$ on $\left[0, T_{\beta}\right]$. Lemma 1 shows that

$$
m\left(x, t+t_{0}\right) \geq w(x, t) \text { on } \mathbb{R}^{+} \times\left[0, T_{\beta}\right],
$$


and since

$$
w\left(x, T_{\beta}\right) \geq q_{\beta}(x-1) \text { on }\left[1,1+\ell_{\beta}\right]
$$

we get

$$
m\left(x, T_{\beta}+t_{0}\right) \geq q_{\beta}(x-1) \quad \text { on } \quad\left(1, \ell_{\beta}+1\right) .
$$

Step 4: Let $u$ be the solution of

$$
\begin{gathered}
u_{t}=\Delta u+f(u) \text { in } \mathbb{R}^{+} \times \mathbb{R}^{+} \\
u(x, 0)= \begin{cases}q_{\beta}(x-1) & \text { in }\left(1,1+\ell_{\beta}\right) \\
0 & \text { elsewhere }\end{cases} \\
u(0, t)=\chi(t)
\end{gathered}
$$

Lemma 2 shows that $\lim _{t \rightarrow \infty} u(x, t)=\bar{\tau}(x)$ where $\bar{\tau}$ is the smallest nonnegative solution of $\tau^{\prime \prime}+f(\tau)=0$ which satisfies $\bar{\tau}(0) \geq \lim _{t \rightarrow \infty} \chi(t)=\beta$ and $\bar{\tau}(x)>q_{\beta}(x-1)$ on $\left(1,1+\ell_{\beta}\right)$ and since $m\left(x, T_{\beta}+t_{0}\right) \geq q_{\beta}(x-1)$ in $\left(1,1+\ell_{\beta}\right)$, Lemma 1 shows that $m(x, t) \geq u(x, t)$.

Step 5: Finally,

$$
\liminf _{t \rightarrow \infty} m(x, t) \geq \bar{\tau}(x)
$$

which implies

$$
b \geq \lim _{x \rightarrow \infty} \liminf _{t \rightarrow \infty} m(x, t) \geq \lim \bar{\tau}(x)
$$

and since

$$
\bar{\tau}\left(\frac{1}{2} \ell_{\beta}+1\right) \geq q_{\beta}\left(\frac{\ell_{\beta}}{2}\right)=\beta>\kappa
$$

the same argument as at step 2 shows that $\bar{\tau}$ is increasing and tends to $b$ as $x$ tends to infinity. This allows to conclude that

$$
\lim _{x \rightarrow \infty} \liminf _{t \rightarrow \infty} m(x, t)=b .
$$

Theorems 3 and 4 not only show that the state $m \equiv 0$ is unstable under compactly supported perturbations, but that $m \equiv b$ is stable under such perturbations. Theorems 2, 3 and 4 bring to light a threshold phenomenon: a disturbance of bounded support of the state $m \equiv 0$ which is sufficiently large on a sufficiently large interval grows to $b$, while a not sufficiently large perturbation tends to vanish.

The reader has to note that sufficient conditions that allow the convergence to the steady state $b$ are either given on the initial condition, or on the boundary condition. One can imagine that if we have a boundary condition and an initial condition which do not fulfill the conditions (3.12), (3.16) and (3.15), but where the initial condition is 'close enough' to the positive boundary we can observe a convergence to the steady state $b$, where 'close enough' has to be understood in the sense that we need enough mass in a small area at the same time. This will be investigated numerically at Section 5.1. 


\section{Wave Propagation}

In this section we look at solutions of (3.8) as wave fronts. To this end we introduce the coordinate

$$
\xi:=x-c t, \quad \text { with } \quad c>0
$$

and the new function

$$
v(\xi, t):=m(\xi+c t, t)
$$

Then the set $\mathbb{R}^{+} \times \mathbb{R}^{+}$is mapped onto the set $\{(\xi, t): \xi>-c t, t>0\}$. Equation (3.8) becomes

$$
v_{t}=\Delta \xi v+c v_{\xi}+f(v)
$$

Note that both Lemmas 1 and 2 can be extended to this equation. In particular Lemma 2 is extended as follows.

Lemma 4 Let $q: \mathbb{R}^{+} \longrightarrow[0, b]$ be a solution of the equation $q^{\prime \prime}+c q+f(q)=0$ in $\left(\xi_{1}, \xi_{2}\right)$ with $\xi_{1}>0$, and let $q\left(\xi_{1}\right)=q\left(\xi_{2}\right)=0$. Let $v(\xi, t)$ denote the solution of the initial-boundary value problem

$$
\begin{gathered}
v_{t}=\Delta v+c v_{\xi}+f(v) \text { in }\{(\xi, t): \xi>-c t, t>0\}, \\
v(\xi, 0)=\left\{\begin{array}{cc}
q(\xi) \text { in } \quad\left(\xi_{1}, \xi_{2}\right) \\
0 \text { in }\{(\xi, t): \xi>-c t, t>0\} \backslash\left(\xi_{1}, \xi_{2}\right)
\end{array}\right. \\
v(-c t, t)=0 \text { in } \mathbb{R}^{+} .
\end{gathered}
$$

Then $v$ is nondecreasing in $t$ and

$$
\lim _{t \rightarrow \infty} v(\xi, t)=\tau(\xi)
$$

where $\tau$ is the smallest nonnegative solution of

$$
\tau^{\prime \prime}+c \tau^{\prime}+f(\tau)=0 \text { in } \mathbb{R}^{+}
$$

which satisfies the inequalities

$$
\tau(\xi) \geq q(\xi) \text { in }\left(\xi_{1}, \xi_{2}\right)
$$

We are interested in the steady-state equation corresponding to equation (4.1), which is

$$
q^{\prime \prime}+c q^{\prime}+f(q)=0
$$

that can be written as a first order system

$$
\begin{aligned}
q^{\prime} & =p \\
p^{\prime} & =-c p-f(q) .
\end{aligned}
$$

The functions $p(\xi)$ and $q(\xi)$ corresponding to a solution of (4.3) give a trajectory in the phase plane. Such a trajectory satisfies

$$
\frac{\mathrm{d} p}{\mathrm{~d} q}=-c-\frac{f(q)}{p}, \text { for } \quad p \neq 0
$$




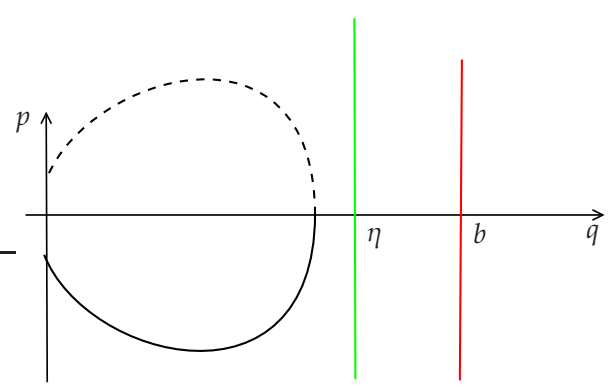

Fig. 6 Trajectories for small value of $c$. The upper line is deduced by symmetry.

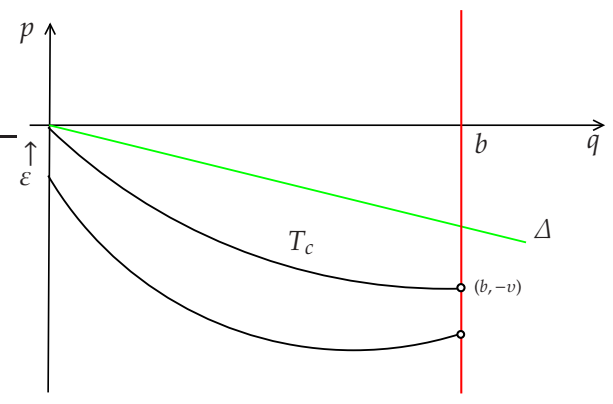

Fig. 7 Trajectories and the line $\Delta$ defined at (4.5) for large enough $c$.

In the case $c=0$. Each trajectory in the phase plane satisfies an equation of the form

$$
\frac{1}{2} p^{2}+F(q)=C
$$

Let fix $\eta, \kappa<\eta<b$. We have $F(\eta)>0$. Then for any $\varepsilon, 0<\varepsilon<\sqrt{2 F(\eta)}$ the trajectory through $(0,-\varepsilon)$ satisfies

$$
F(\eta)>\frac{1}{2} \varepsilon^{2}+F(0)=\frac{1}{2} p^{2}+F(q)
$$

which implies that the trajectory is confined to the strip $0 \leq q<\eta$. Hence by symmetry the trajectory crosses the $p=0$ axis at $(\alpha, 0)$ and the $q=0$ axis at $(0, \varepsilon)$. By continuity this holds true for small velocities: there exists $\tilde{c}(\varepsilon)$ such that for all $c \in[0, \tilde{c}(\varepsilon))$ the trajectory crosses the $p=0$ axis at $(\alpha, 0)$ and the $q=0$ axis at $(0, \bar{\varepsilon})$ with $\bar{\varepsilon}>0$. See Figure 6 .

The case $c>0$. Since in our case we always have $c^{2}>4 f^{\prime}(0)$ the eigenvalues of the Jacobian matrix of System (4.3) are real, there is no spiral point in $(0,0)$ and there exists one non trivial trajectory through $(0,0)$. In fact, if we seek a linear solution around the origin $p=\alpha q$ we find out that $\alpha$ should satisfy $\alpha^{2}+c \alpha+f^{\prime}(0)=0$ which has a real solution if $c^{2}>4 f^{\prime}(0)$, which holds here since $f^{\prime}(0)<0$. The unique trajectory through $(0,-\varepsilon)$ with $\varepsilon>0$, cannot cross the trajectory that goes to the origin (Cauchy property). Hence if we take the limit trajectory as $\varepsilon$ decreases to 0 we obtain a non trivial trajectory going to the origin. We denote by $T_{c}$ this extremal trajectory.

We define

$$
\sigma:=\sup _{u \in[0, b]} \frac{f(u)}{u}
$$


Note that $\sigma \geq f^{\prime}(0)$ and $f(u) \leq \sigma u$ for $u \in[0, b]$. It follows that for any trajectory $T$,

$$
\frac{\mathrm{d} p}{\mathrm{~d} q} \leq-c-\sigma \frac{q}{p}
$$

at any point of $T$ where $q \in[0, b]$ and $p<0$. Furthermore

$$
\Delta: \quad p=-\frac{1}{2}\left(c+\sqrt{c^{2}-4 \sigma}\right) q
$$

is a solution of

$$
\frac{\mathrm{d} p}{\mathrm{~d} q}=-c-\sigma \frac{q}{p}
$$

and thus the trajectory through $(0,-\varepsilon)$, with $\varepsilon>0$ lies under the line defined by (4.5). As $\varepsilon$ tends to 0 we note that $T_{c}$ is bounded above by the line defined by (4.5) and in particular connects the origin to a point of the shape $(b,-v)$ with $v>0$. (See Figure 7.) Thus,

$$
c^{*}:=\inf \left\{c \geq 0: \exists v>0 \text { s.t. }(b,-v) \in T_{c}\right\}
$$

is well-defined. In what follows, we show that $c^{*}$ is the asymptotic speed of propagation associated to the equation (2.1) and that the limit trajectory $T_{c^{*}}$ connects the origin to $(b, 0)$.

Theorem 5 The trajectory corresponding to the asymptotic speed of propagation, $T_{c^{*}}$, goes from $(b, 0)$ to $(0,0)$ in the lower phase plane.

Proof We have already shown that the trajectory $T_{c^{*}}$ exists and lies in the half-strip $q \in[0, b], p \leq 0$ at least in a neighbourhood of the origin. $T_{c^{*}}$ is minimal in the sense that there is no other trajectory which reaches the origin and lies below $T_{C^{*}}$.

Step 1: We first show that $T_{c^{*}}$ must cross the $(p=0)$ axis at some point $\eta$ with $f(\eta)=0$. Suppose that $T_{c^{*}}$ does not cross the $(p=0)$ axis, then it holds true for slightly smaller values of $c$ which contradicts the definition of $c^{*}$. Hence $T_{c^{*}}$ crosses the $(p=0)$ axis at some point $(\eta, 0)$ with $\eta \in(0, b]$. Suppose $f(\eta) \neq 0$ then necessarily $f(\eta)>0$ : in fact $T_{c^{*}}$ must go in the negative $q$-direction as $p<0$ (see (4.3)) which implies that $-c-\frac{f(q)}{p}$ should be positive as $(q, p)$ tends to $(\eta, 0)$ which is true only if $f(\eta)>0$. Then there exists $\eta_{1}$ such that $f\left(\eta_{1}\right)>0$ and $\eta_{1}>\eta$. The part of the trajectory $T$, that goes through $\left(\eta_{1}, 0\right)$ lies below $T_{c^{*}}$ and crosses the $(q=0)$ axis at $(0,-v)$ with $v>0$. By continuity, $T_{c}$ for $c$ slightly greater than $c^{*}$, will be bounded from below by $T$ and can not cross the half line $q=b, p<0$ which contradicts the definition of $c^{*}$. (See Figure 8.) We conclude that $T_{c^{*}}$ hits the axis $(p=0)$ at a point $(\eta, 0)$ where $f(\eta)=0$.

Step 2: Let us show now that $\eta=b$. Since the trajectory nearby the origin lies in the half-plane $p<0$ and, according to (4.4), if $f(q)<0$ then the slope of $T_{c^{*}}$ is negative and the trajectory can not cross the $(p=0)$ axis at a zero of $f$ which is the right end point of an interval where $f$ is negative. Thus $T_{c^{*}}$ must hit the $(p=0)$ axis at $(b, 0)$.

Theorem 6 Let $m(x, t): \mathbb{R}^{+} \times \mathbb{R}^{+} \mapsto[0, b]$ be the solution of (3.8). The asymptotic velocity $c^{*}$ is defined as in (4.6).

a) Then for any $c>c^{*}$ and any real $\xi$

$$
\lim _{t \rightarrow \infty} m(\xi+c t, t)=0 .
$$

b) If $\lim _{x \rightarrow \infty} \liminf _{t \rightarrow \infty} m(x, t)=b$, then for any $c \in\left(0, c^{*}\right)$ and any real $\xi$

$$
\lim _{t \rightarrow \infty} m(\xi+c t, t)=b .
$$




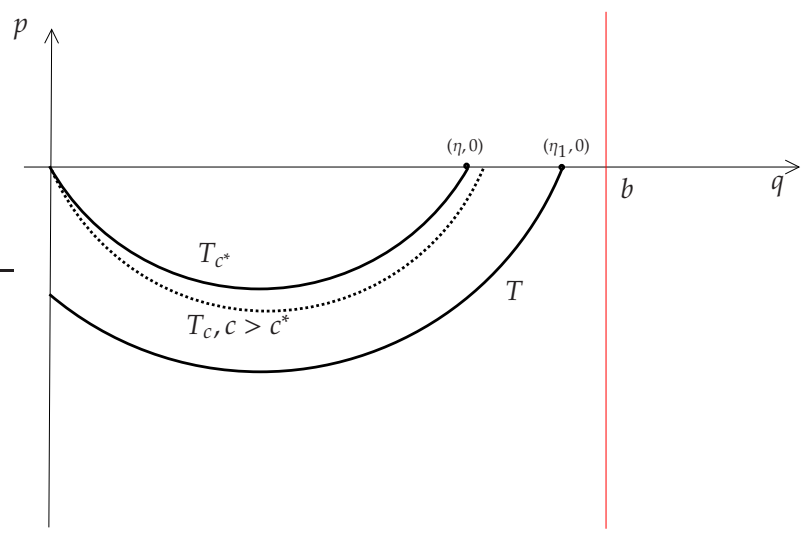

Fig. 8 If $f(\eta) \neq 0$

Proof Let us prove point a).

Step 1: Let $q_{c}(x)$ denote the solution of the steady state equation (4.2) in $\mathbb{R}^{+}$which corresponds to the trajectory

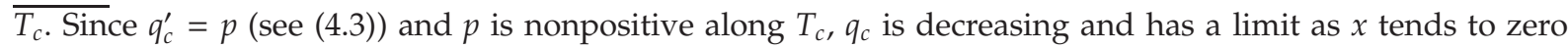
which is necessarily 0 . Let us define $w=b-m$. To prove the result we are going to show that the limit of $w$ as $t$ tends to infinity is larger than $b$. The function $w$ satisfies

$$
w_{t}=\Delta w-f(b-w) .
$$

We apply the extension of Lemma 1 and 2 with $q=b-q_{c}\left(x-x_{0}\right)$ in $\left(x_{0}, \infty\right)$. First Lemma 1 shows that

$$
b-q_{c}\left(\xi-x_{0}\right) \leq w(\xi+c t, t) \quad \text { in } \quad\left(x_{0}, \infty\right) \times \mathbb{R}^{+} .
$$

Let us define then the function $v$ such that

$$
v_{t}=\Delta_{\xi} v+c v_{\xi}+f(v) \quad \text { in } \quad\{(\xi, t): \xi>-c t, t>0\}
$$

and

$$
b-v(\xi, 0)= \begin{cases}b-q_{c}\left(\xi-x_{0}\right) & \text { in }\left(x_{0}, \infty\right) \\ 0 & \text { in } \mathbb{R} \backslash\left(x_{0}, \infty\right)\end{cases}
$$

Again using Lemma 1, we get

$$
b-v(\xi, t) \leq w(\xi+c t, t) \quad \text { in } \quad\left(x_{0}, \infty\right) \times \mathbb{R}^{+} .
$$

Futhermore, Lemma 4 shows that

$$
\lim _{t \rightarrow \infty} v(\xi, t)=\tau(\xi),
$$

where $b-\tau(\xi)$ is the smallest nonnegative function of

$$
\tau^{\prime \prime}+c \tau^{\prime}+f(\tau)=0 \quad \text { in } \quad \mathbb{R},
$$

which satifies the inequality

$$
b-\tau(\xi) \geq b-q_{c}\left(\xi-x_{0}\right) \text { in }\left(x_{0}, \infty\right) .
$$

Finally, it proves that

$$
\liminf _{t \rightarrow \infty}(b-m(\xi+c t, t)) \geq b-\tau(\xi) .
$$


Step 2: We must show now that $\tau(\xi) \equiv 0$. For any $c>0$ the slope of trajectory $T_{c}$ is $s^{-}$at the origin, where

$$
s^{-}=\frac{1}{2}\left(-c-\sqrt{c^{2}-4 f^{\prime}(0)}\right) \text {. }
$$

Moreover, $T_{c}$ is the unique trajectory with this slope at the origin and any other trajectory which approaches the origin with $q>0$ must have the slope

$$
s^{+}=\frac{1}{2}\left(-c+\sqrt{c^{2}-4 f^{\prime}(0)}\right)
$$

at the origin.

Since $c>c^{*}$, the trajectory $T_{c}$ stays in the half-plane $p<0$ for $q \in(0, b]$ and contains a point $(b,-v)$ with $v>0$. Suppose that $\tau(\xi) \not \equiv 0$. Then the corresponding trajectory has either the slope $s^{+}$or $s^{-}$at the origin. But it follows from (4.7) that the trajectory $T$ should stay on the left of $T_{c}$ in particular at the origin. Since $T_{c}$ has the slope $s^{-}$, we deduce that necessarily $T$ has also the slope $s^{-}$at $(0,0)$ and by uniqueness $T \equiv T_{c}$. This leads us to a contradiction because there exists $\zeta \in \mathbb{R}$ such that $\tau(\zeta)=b$ and $\tau^{\prime}(\zeta)=-v<0$. Hence $\tau(\xi)>b$ for some $\xi<\zeta$ and this contradicts the nonnegativeness of the function $b-\tau$. We conclude that $\tau \equiv 0$.

Step 3: The equation (4.8) and the positiveness of $m$ prove the first part of the theorem.

Let us now prove point $b)$.

Step 1: There exists a trajectory $T$ that goes from a point on the positive $p$-axis to a point $(\beta, 0)$ and from there to a point on the negative $p$-axis.

The proof of theorem 5 shows that $T_{c^{*}}$ goes from $(b, 0)$ to $(0,0)$ in the lower phase plane. Consider any $c<c^{*}$. Because of equation (4.4), the trajectory $T_{c}$ lies above $T_{c^{*}}$ and crosses the $q$-axis at a point $(\eta, 0)$, with $\eta \in(0, b)$. Then if $\beta \in(\kappa, b)$ (thus $\left.\int_{0}^{\beta} f>0\right)$, with $\kappa$ defined in (3.5), the lower part of the trajectory $T$ through $(\beta, 0)$ stays below $T_{c}$. Therefore, $T$ goes to the negative $p$-axis. Since $f$ is postive nearby $u=\beta$, the slope of $T$ is negative in the upper half-plane in a neighborhood of $(\beta, 0)$. Moreover, the slope is bounded from below when $p$ is bounded away from zero. Therefore the trajectory does not go to infinity. From equation (4.4), we get:

$$
\frac{\mathrm{d} p}{\mathrm{~d} q} \leq-\frac{f(q)}{p}
$$

which leads to the following inequality:

$$
\int_{q_{0}}^{\beta} f(q) \mathrm{d} q \leq p 2\left(q_{0}\right), \quad \forall q_{0} \in(0, \beta), p>0 .
$$

As a consequence the trajectory $T$ cannot cross again the $q$-axis and necessarily it crosses the positive $p$-axis.

Step 2 : We have shown that for each $c \in\left(0, c^{*}\right)$ there is a trajectory $T$ which connects the positive $p$-axis to the negative $p$-axis. $T$ crosses the $q$-axis at a point $(\beta, 0)$ with $\beta \in(\kappa, b)$, and lies in the strip $q \in[0, \beta]$. Let $q_{\beta}$ be the corresponding solution of $q^{\prime \prime}+c q^{\prime}+f(q)=0$ for which $q_{\beta}(0)=0, q_{\beta}^{\prime}(0)>0$. This solution is positive on a finite interval $(0, \delta)$ and $q_{\beta}(\delta)=0$. Moreover, $q_{\beta}(x) \leq \beta<b$. Since we suppose that $\lim _{x \rightarrow \infty} \liminf _{t \rightarrow \infty} m(x, t)=b$, uniformly on each interval, there is a time $\theta$ and a constant $A$ so that

$$
m(x, \theta) \geq \beta \geq q_{\beta}(x) \quad \text { on } \quad[A, A+\delta]
$$

Recalling that $v(\xi, t)=m(\xi+c t, t)$, the previous property can be writen as follows:

$$
v(\xi, \theta) \geq \beta \geq q_{\beta}(\xi) \quad \text { on } \quad[A-c \theta, A+\delta-c \theta] .
$$


We apply Lemma 4 with $q(\xi)=q_{\beta}(\xi)$ : let define

$$
\begin{gathered}
\bar{v}_{t}=\Delta \bar{v}+c \bar{v}_{\xi}+f(\bar{v}), \quad \text { in }\{(\xi, t): \xi>-c t, t>0\} \\
\bar{v}(\xi, \theta)= \begin{cases}q_{\beta}(\xi) & \text { on }(A-c \theta, A+\delta-c \theta) \\
0 & \text { on } \mathbb{R} \backslash(A-c \theta, A+\delta-c \theta) .\end{cases}
\end{gathered}
$$

Then

$$
\lim _{t \rightarrow \infty} \bar{v}(\xi, t)=\tau(\xi)
$$

uniformly on each bounded interval, where $\tau$ is the smallest nonnegative function of

$$
\tau^{\prime \prime}+c \tau^{\prime}+f(\tau)=0
$$

on $\mathbb{R}$ which satisfies $\tau(\xi) \geq q_{\beta}(\xi)$ on $(A-c \theta, A+\delta-c \theta)$. Furthermore, note that according to the extension of Lemma 1 we have

$$
v(\xi, t) \geq \bar{v}(\xi, t)
$$

Step 3 : We show now that $\tau \equiv b$. Suppose that the trajectory corresponding to $\tau$, denoted by $T_{\tau}$, crosses the $\overline{q \text {-axis }}$ at one point $\left(\eta_{\tau}, 0\right)$, with $\beta \leq \eta_{\tau} \leq b$. Then similar arguments as in Step 1 show that $T_{\tau}$ connects the positive $p$-axis to the negative $p$-axis. Thus there exists $\xi_{0}$ such that $\tau\left(\xi_{0}\right)=0$ and $\tau^{\prime}\left(\xi_{0}\right)>0$ which is in contradiction with $\tau$ being nonnegative. Thus, $\tau \equiv b$, and since we suppose $m(x, t) \leq b$, we end up with:

$$
\lim _{t \rightarrow \infty} m(\xi+c t, t)=\lim _{t \rightarrow \infty} v(\xi, t)=b .
$$

\section{Numerical Study of the Wave Propagation}

In this last part of the paper we describe numerical simulations of the system at Section 5.1 exploring the behavior of the solution for several boundary and initial conditions. In Section 5.2 we compute numerically the asymptotic velocity $c^{*}$, and describe its dependence on the parameter $\alpha$.

\subsection{Approximation of the solution}

We compute the solution using a Crank-Nicolson scheme. It is a scheme of order 2 in space and time with no stability condition. Let us fix in this part $\alpha=4>\alpha_{c}$ defined at (3.3).

Following Theorem 1 the appearance of a front should depend on the value of $\sup _{t} \psi(t)$. The value of $\kappa$, which itself depends on $\alpha$ as defined in (3.5), has a significant influence on the behavior of the system. In Figure 9, we plot the graph of the function $\kappa: \alpha \mapsto \kappa(\alpha)$. We observe that this function is decreasing: $\kappa$ increases with the degradation term (which is proportional to $1 / \alpha$ ). This is relevant biologically : the amount of mass needed to observe a wave front is reduced when the degradation rate goes down. For the simulation of the wave front formation, we choose $\alpha=4$, which leads to $\kappa=1.24$. In Figure 10, we illustrate the threshold phenomena stated in Theorem 1.

Now Theorem 4 suggests that even when the hypothesis $\sup _{t} \psi(t)>\kappa$ is fulfilled, the support of $\psi$ must be long enough. This is confirmed numerically (see Figure 11).

Theorem 3 states that in case of an homogeneous Dirichlet boundary condition, the initial condition has to be large enough to ensure the appearance of a wave front. In Figure 12, we study the influence of the initial condition. Note that we chose a gaussian initial profile, because from our point of view it is biologically relevant. We observe that the output, i.e. the existence of a wave front or not, should depend on the initial 


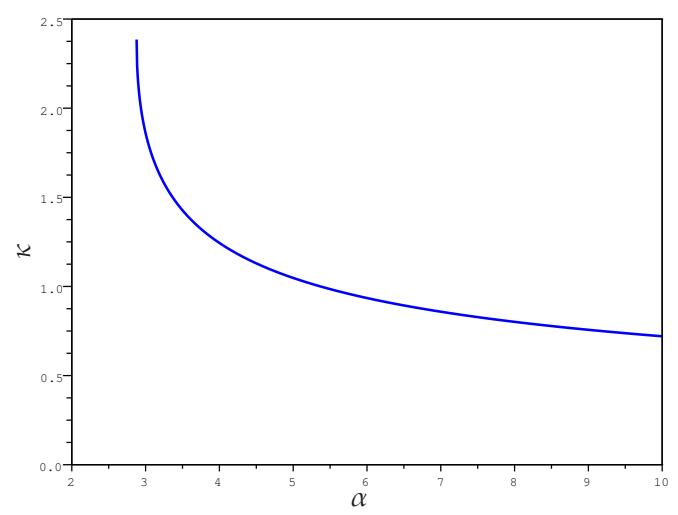

Fig. $9 \kappa: \alpha \mapsto \kappa(\alpha)$

mass. This not totally true, applying some different shape for a constant initial mass we observe different behaviors : the shape of the initial conditions has an influence on the output of the cascade. (See Figure 13 and 14.)

Now, we combine both positive boundary and initial conditions. (See Figure 15.) The reader has to note that there is a delay due to the time needed by the mass located in the boundary to reach the initial condition (compare for instance the two first line of Figure 15). This illustrates in particular our comment on the idea to be 'close enough' at the end of Section 3, page 15. 

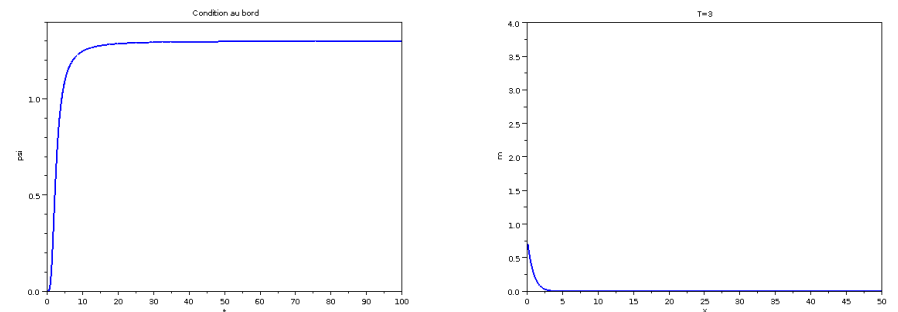

(a) Boundary condition, $\sup _{t} \psi(t)=1.3$

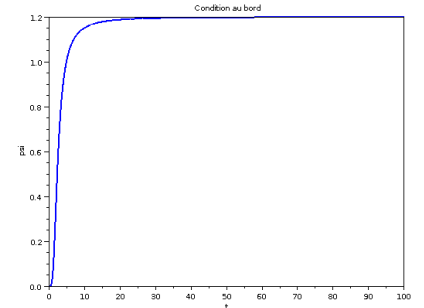

(e) Boundary condition, $\sup _{t} \psi(t)=1.2$

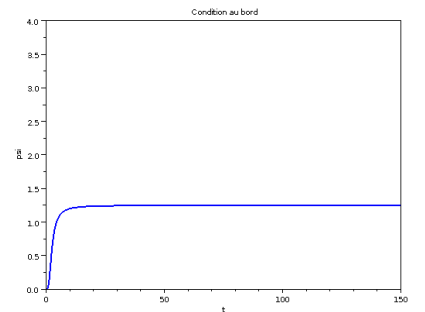

(i) Boundary condition, $\sup _{t} \psi(t)=$ 1.25

Fig. 10 Influence of $\sup _{t} \psi(t)$; here $\psi(t)=\psi_{\max } \frac{(0.5 t)^{4}}{1+(0.5 t)^{2}+(0.5 t)^{4}}, \kappa=1.24$.

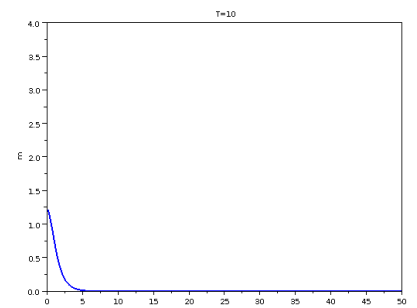

(c) Solution, $T=10$

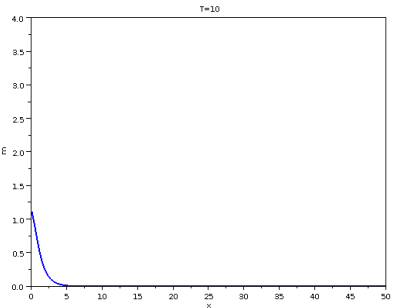

(g) Solution, $T=10$

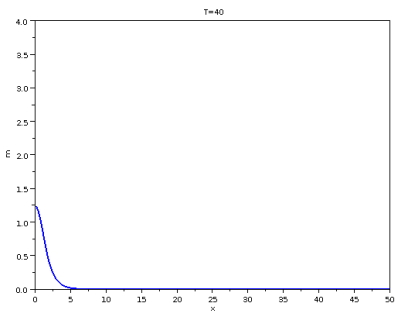

(k) Solution, $T=40$

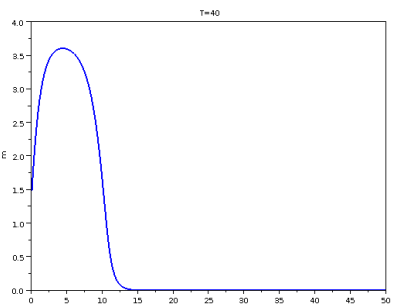

(d) Solution, $T=40$

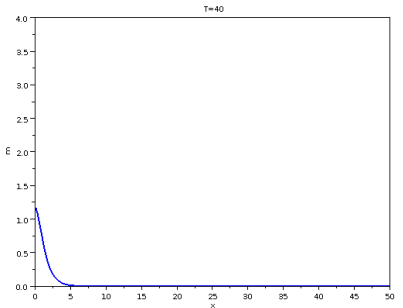

(h) Solution, $T=40$

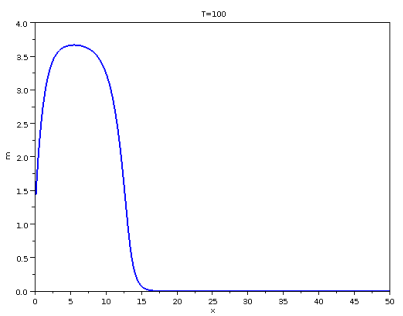

(l) Solution, $T=100$ 


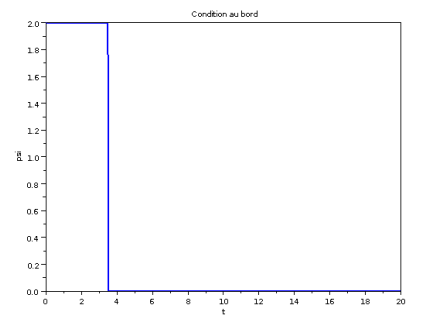

(a) Boundary condition, $\psi(t)=2$ on $(0,4)$

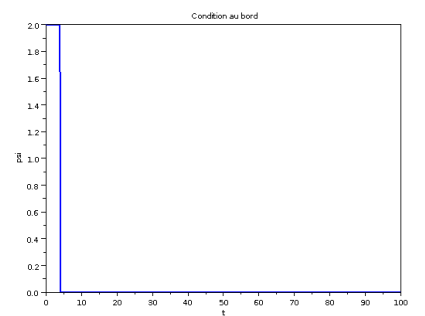

(e) Boundary condition, $\psi(t)=2$ on $(0,3.5)$

Fig. 11 Influence of the length of the support of $\psi, \kappa=1.24$.

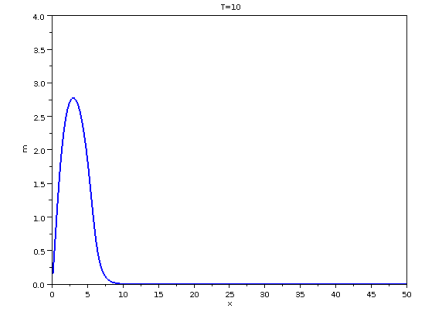

(c) Solution, $T=10$

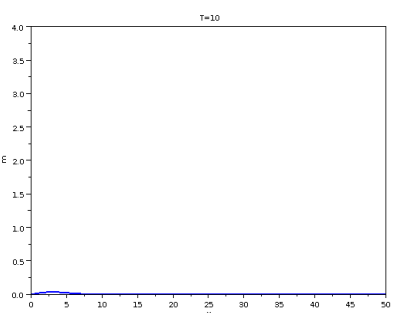

(g) Solution, $T=10$

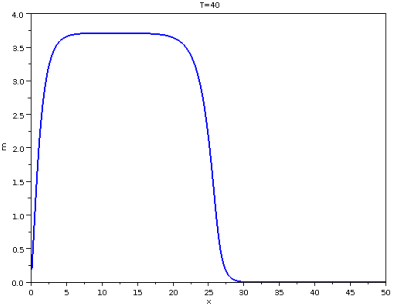

(d) Solution, $T=40$

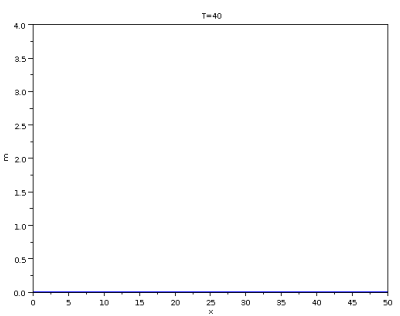

(h) Solution, $T=40$

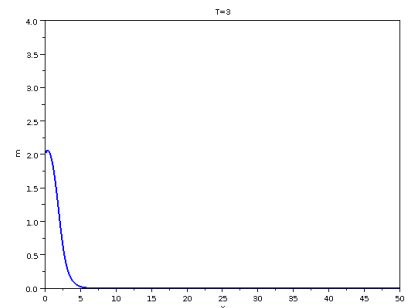

(f) Solution, $T=3$ 


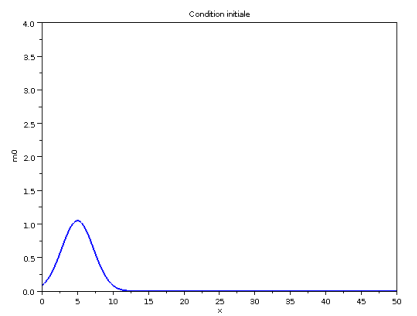

(a) Initial condition, $\left\|m_{0}\right\|=1.05$

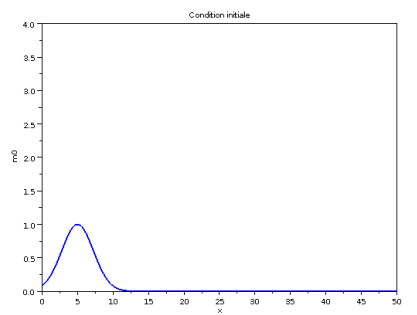

(e) Initial condition, $\left\|m_{0}\right\|=1.0$

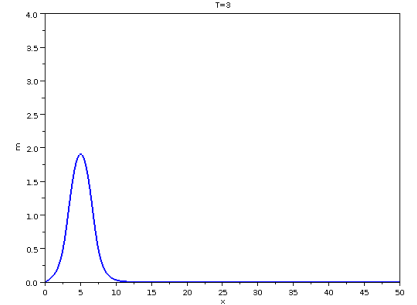

(b) Solution, $T=3$

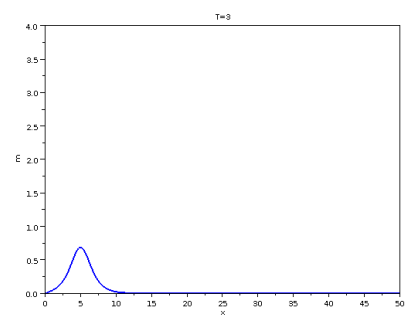

(f) Solution, $T=3$

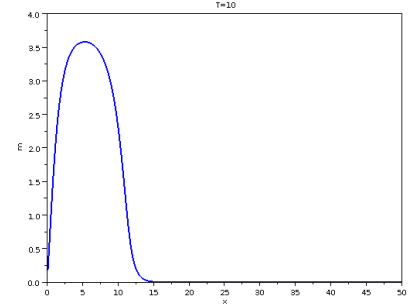

(c) Solution, $T=10$

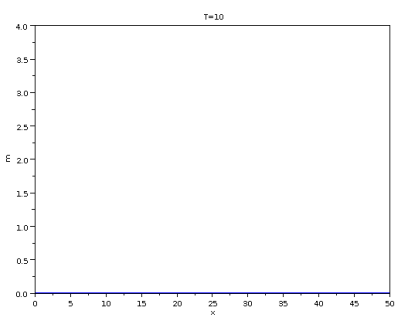

(g) Solution, $T=10$

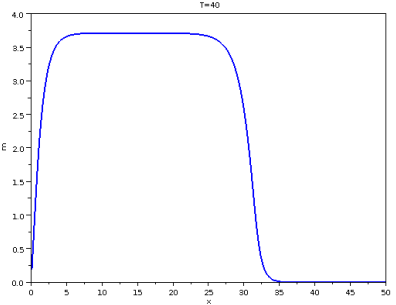

(d) Solution, $T=40$

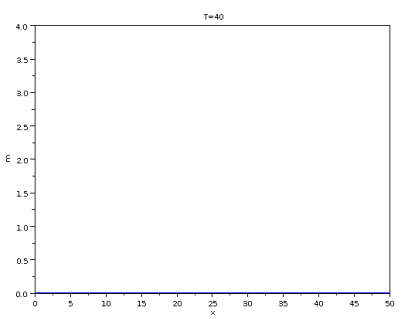

(h) Solution, $T=40$

Fig. 12 Influence of the initial condition, $\psi(t) \equiv 0, \kappa=1.24$. 


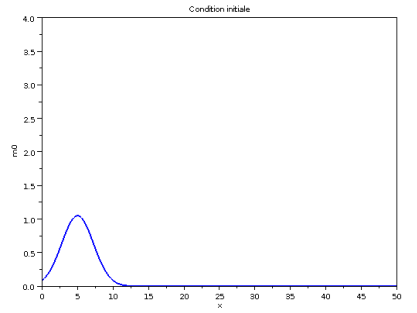

(a) Initial condition, $\int m_{0}=M_{0}^{1}$

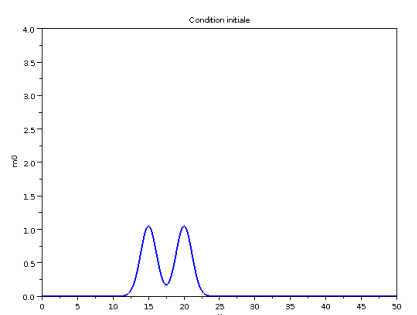

(e) Initial condition, $\int m_{0}=M_{0}^{1}$

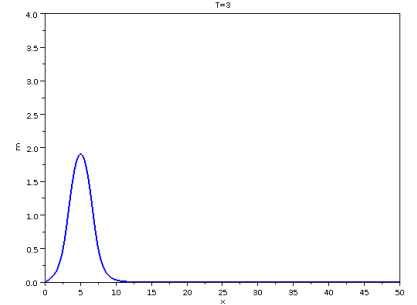

(b) Solution, $T=3$

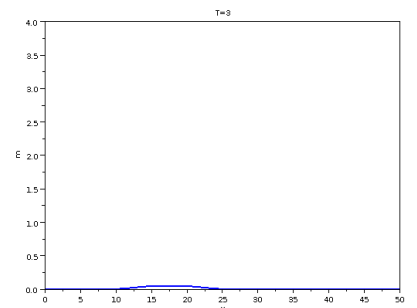

(f) Solution, $T=3$

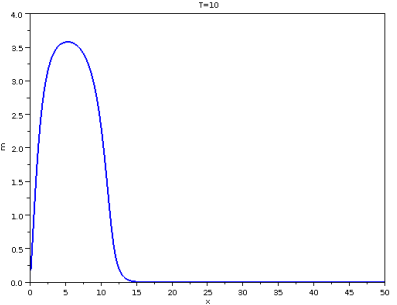

(c) Solution, $T=10$

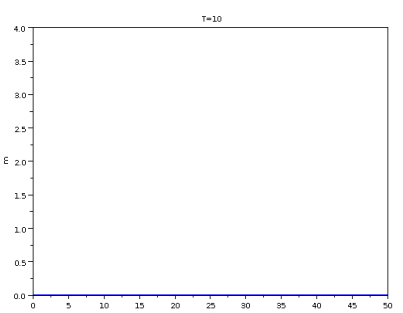

(g) Solution, $T=10$

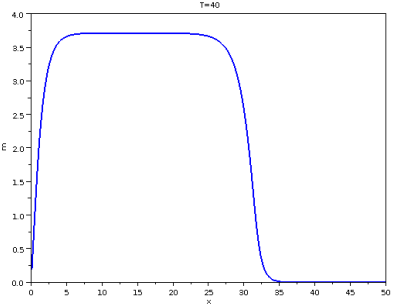

(d) Solution, $T=40$

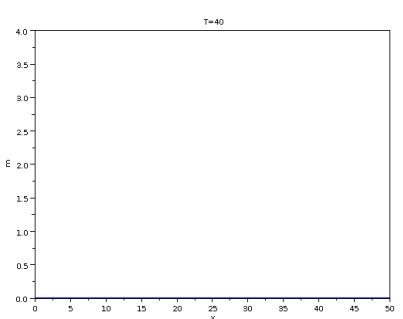

(h) Solution, $T=40$

Fig. 13 Influence of the shape of the initial condition for a given initial mass, $\psi(t) \equiv 0, \kappa=1.24$. 


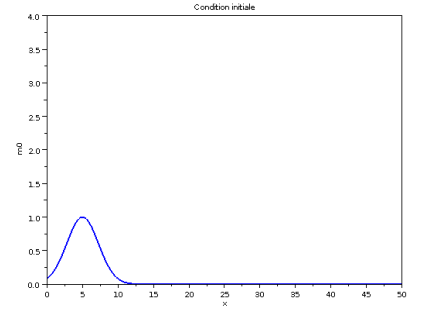

(a) Initial condition, $\int m_{0}=M_{0}^{2}$

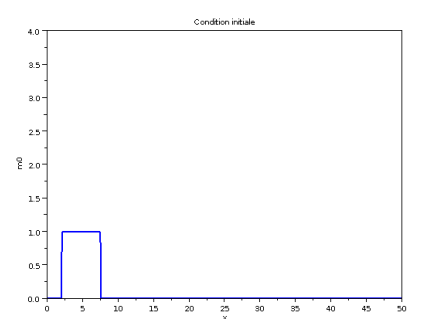

(e) Initial condition, $\int m_{0}=M_{0}^{2}$

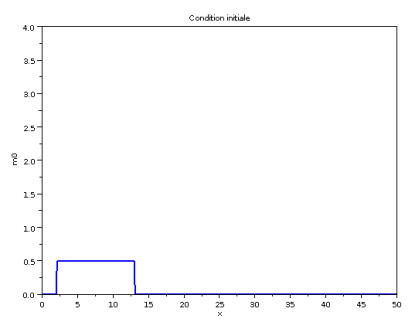

(i) Initial condition, $\int m_{0}=M_{0}^{2}$

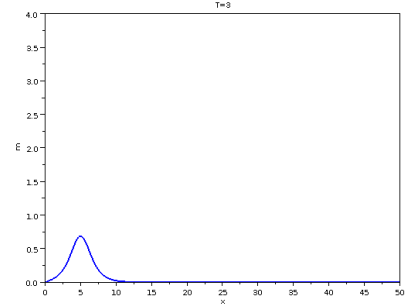

(b) Solution, $T=3$

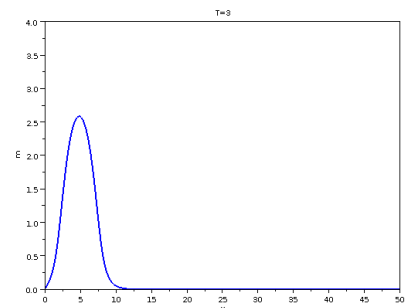

(f) Solution, $T=3$

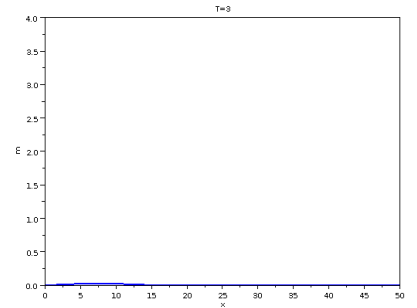

(j) Solution, $T=3$

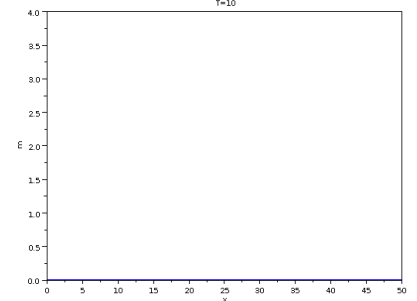

(c) Solution, $T=10$

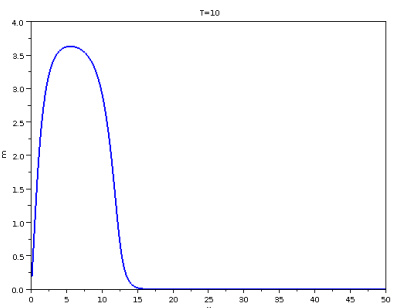

(g) Solution, $T=10$

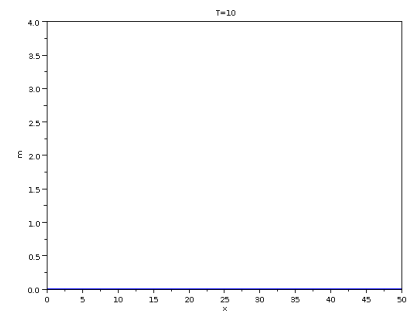

(k) Solution, $T=10$

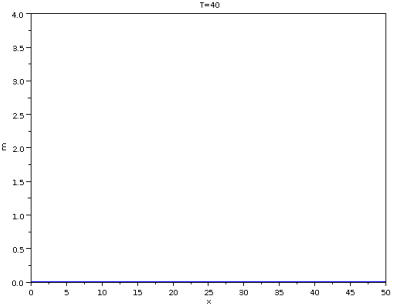

(d) Solution, $T=40$

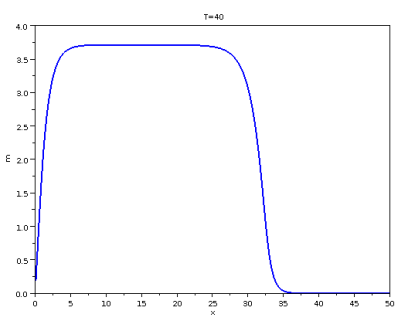

(h) Solution, $T=40$

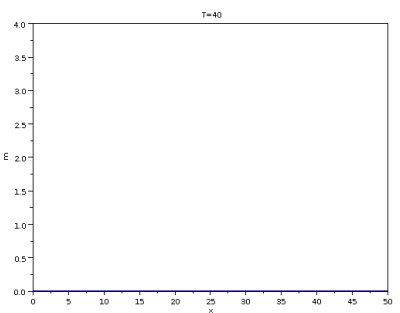

(1) Solution, $T=40$

Fig. 14 Influence of the shape of the initial condition for a given initial mass, $\psi(t) \equiv 0, \kappa=1.24$. 

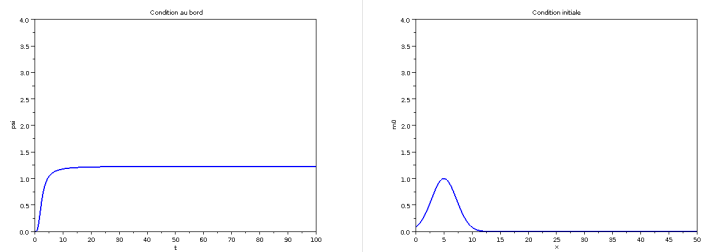

(a) Boundary $\sup _{t} \psi(t)=1.23$

condition, (b) Initial condition, $\left\|m_{0}\right\|=1.0$
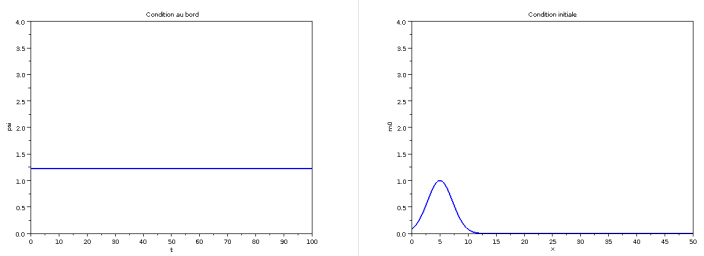

(f) Boundary $\sup _{t} \psi(t)=1.23$

condition, (g) Initial condition, $\left\|m_{0}\right\|=1.0$
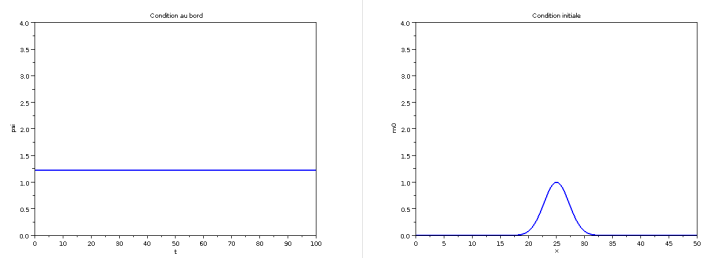

(k) Boundary condition, (l) Initial condition, $\left\|m_{0}\right\|=1$. $\sup _{t} \psi(t)=1.23$

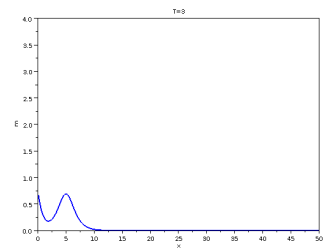

(c) Solution, $T=3$

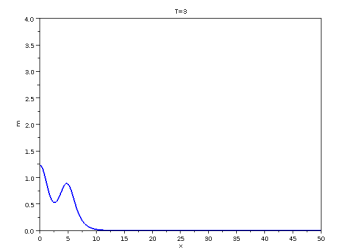

(h) Solution, $T=3$

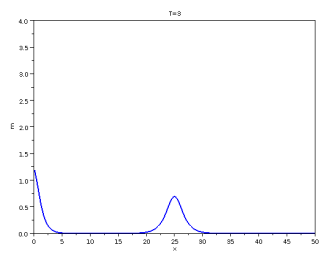

(m) Solution, $T=3$

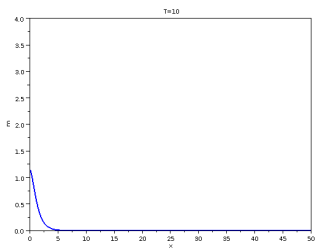

(d) Solution, $T=10$

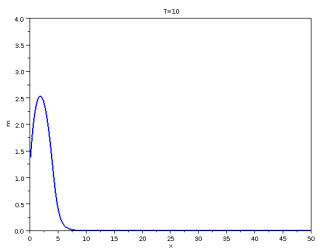

(i) Solution, $T=10$

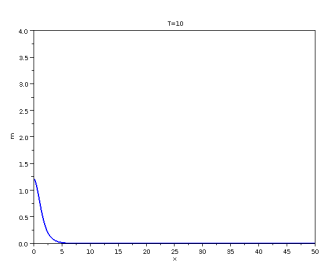

(n) Solution, $T=10$

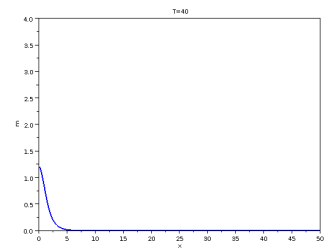

(e) Solution, $T=40$

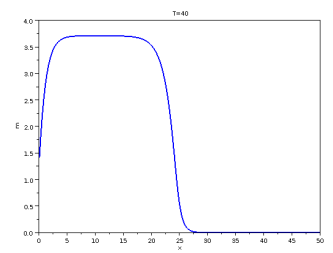

(j) Solution, $T=40$

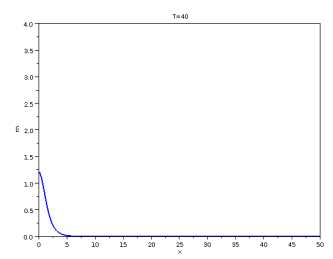

(o) Solution, $T=40$

Fig. 15 Influence of both initial condition and boundary condition. $\kappa=1.24$. 


\subsection{Asymptotic velocity}

\subsubsection{Estimation of the asymptotic velocity}

In this section we want to evaluate numerically the asymptotic velocity defined at (4.6). The method we use is a finite-difference discretization combined to Newton's method.

At first, the trajectories in the phase plane are given by Equation (4.4), but one should note that the righthand side of $(4.4)$ is not defined in $(0,0)$. Nevertheless, we know the slope of the trajectory at the origin (see the proof of Theorem 6 a) Step2). This is why we choose to solve numerically:

$$
\begin{cases}\frac{\mathrm{d} p}{\mathrm{~d} q}=-c^{*}-\frac{f(q)}{p} & \text { if } p \neq 0, \\ \frac{\mathrm{d} p}{\mathrm{~d} q}(0)=\frac{1}{2}\left(-c^{*}-\sqrt{c^{* 2}-4 f^{\prime}(0)}\right) & \\ p(0)=p(b)=0 & \end{cases}
$$

We want to discretize problem (5.1). To this end we use a regular grid

$$
0=q_{0}<q_{1}<\cdots<q_{N}<q_{N+1}=b, \quad \text { with } q_{i+1}-q_{i}=\Delta q=\frac{b}{N+1}, \quad N \in \mathbb{N},
$$

and the following forward 1st-order discretization of the problem (5.1) :

$$
\begin{array}{rlrl}
\frac{p_{1}}{\Delta q} & =\frac{\mathrm{d} p}{\mathrm{~d} q}\left(p_{0}\right)=\frac{1}{2}\left(-c-\sqrt{c^{2}-4 f^{\prime}(0)}\right), & \\
\frac{p_{i+1}-p_{i}}{\Delta q} & =\frac{\mathrm{d} p}{\mathrm{~d} q}\left(p_{i}\right)=-c-\frac{f\left(q_{i}\right)}{p_{i}} & \text { for } i=1 \ldots N-1, \\
\frac{-p_{N}}{\Delta q} & =\frac{\mathrm{d} p}{\mathrm{~d} q}\left(p_{N}\right)=-c-\frac{f\left(q_{N}\right)}{p_{N}} .
\end{array}
$$

This leads to the following non linear system:

$$
A P=B,
$$

where

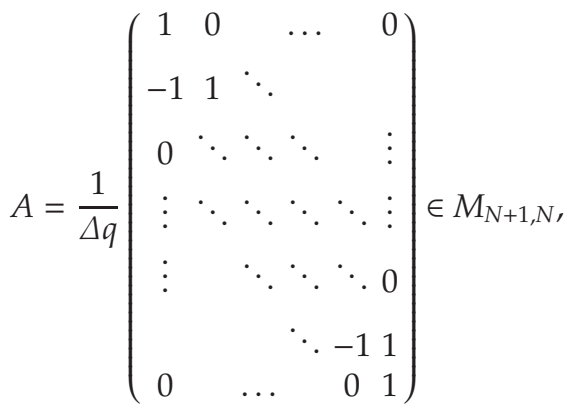

$$
\begin{aligned}
& P=\left(\begin{array}{c}
p_{1} \\
p_{2} \\
\vdots \\
\vdots \\
\vdots \\
p_{N}
\end{array}\right) \in M_{N, 1}
\end{aligned}
$$




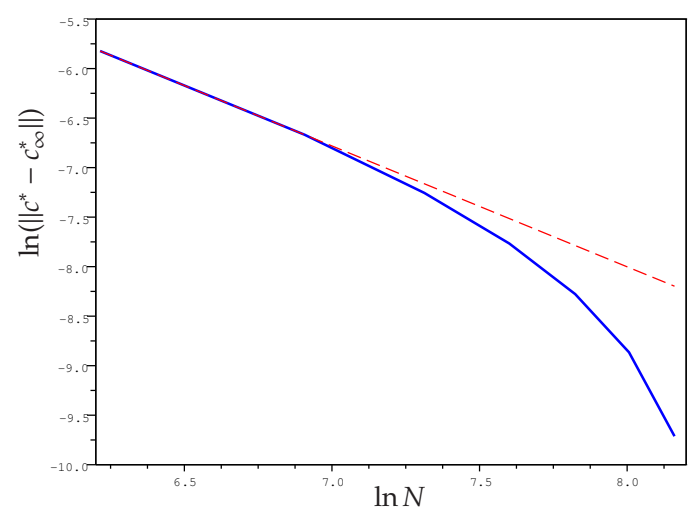

Fig. 16 Convergence to the asymptotic velocity with respect to the size of the grid.

and

$$
B\left(P, c^{*}\right)=\left(\begin{array}{c}
\frac{1}{2}\left(-c^{*}-\sqrt{c^{* 2}-4 f^{\prime}(0)}\right) \\
-c^{*}-\frac{f\left(q_{1}\right)}{p_{1}} \\
-c^{*}-\frac{f\left(q_{2}\right)}{p_{2}} \\
\vdots \\
\vdots \\
\vdots \\
-c^{*}-\frac{f\left(q_{N}\right)}{p_{N}}
\end{array}\right) \in M_{N+1,1}
$$

Note this system is not square. To compute the solution $\left(c^{*}, P\right)$ solution of $(5.1)$ we use a Newton procedure combined with a dichotomy procedure to initialize reasonably well. Because the finite-difference discretization we use is of order 1, at the convergence of the Newton method, which is quadratic by the way, we get an error of order $\Delta q$. To confirm this convergence rate, we plot numerically the logarithm of the error as a function of the logarithm of the size of the mesh. (See Figure. 16.) Using a linear regression, we find out a numerical order of convergence of $r_{\text {num }}=1.22$.

\subsubsection{Dependency of the asymptotic velocity on the parameter $\alpha$}

At Figure 17, we plot the asymptotic velocity as a function of $\alpha$. The velocity is increasing with $\alpha$, this is relevant biologically: the asymptotic velocity goes up when the degradation is reduced. 


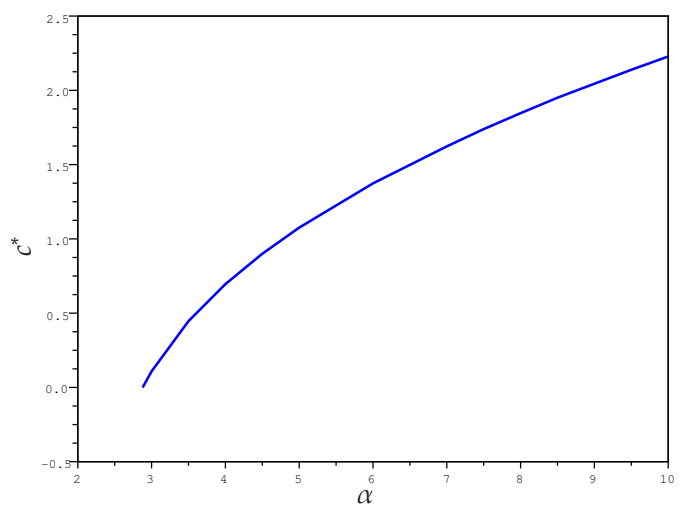

Fig. 17 Dependency of the asymptotic velocity on the parameter $\alpha$.

\subsection{Influence of the Hill exponent}

We want now to compare the results for several values of the Hill exponent $h$. Recall that until now, we have been working with $h=4$, considered as the typical Hill exponent for the MAPK cascade. For $h=2$ the non-linearity (see (2.2)) reads as follows:

$$
z_{\alpha}(m)=\frac{\alpha m^{2}}{m^{2}+1}
$$

A Hill exponent equal to 2 appears in other biological models such as the calcium-stimulated-calcium-release mechamism which happens from calcium sites on the membrane enclosing certain fertilized amphibian eggs ([8]). For more details about the Hill exponent see also [3]. Cases $h=2$ and $h=4$ are both biologically relevant. To complete our simulations we choose to test the case $h=6$ with the non-linearity defined as follows:

$$
z_{\alpha}(m)=\frac{\alpha m^{6}}{m^{6}+m^{3}+1}
$$

Figure 21 displays the shape of the functions $m \mapsto z_{\alpha}(m)$ for the three values of the Hill exponent $h=2, h=4$ and $h=6$. As $h$ increases, the slope is steeper. It would be tempting to correlate the steepness of these curves to the properties of the wave front: velocity of the wave front and speed of the change of state can be expected to vary monotonically with $h$ (typically, the smaller $h$, that is when the slope is less steep, the slower the wave front would be the naive belief). We shall see that such a deduction is erroneous.

Firstly, we plot in Figure 18 the behavior of $\kappa$ as a function of $\alpha$ for $h=2, h=4$ and $h=6$. As one can see, the value of $\kappa$ is lower for $h=2$ than for $h=4$. This means that the amount of mass needed to trigger the wave front is lower for $h=2$ than for $h=4$. Isolating this property is important since checking this consequence of the modeling could be accessible to experiments and the comparison would help in validating the proposed equation. The results obtained with for $h=6$ however indicate that $\kappa$ is not a monotone function of $h$.

Secondly, in Figure 19 we plot the asymptotic velocity as a function of $\alpha$ for $h=2, h=4$ and $h=6$. What could be surprising is that the asymptotic velocity $c^{*}$, which is the speed of signal propagation, is lower for $h=4$ than for $h=2$. The asymptotic velocity is not monotone with respect to $h$.

Thirdly, to precise the comparison for different Hill exponents we plot in Figure 20 the time it takes, when the asymptotic velocity is reached, to switch from the stationary state $m \equiv 0$ to $m \equiv b$. To this end we define the quantity $\Delta$ as follows:

$$
\Delta=z_{\alpha}^{-1}(0.99 \cdot \alpha)-z_{\alpha}^{-1}(0.01 \cdot \alpha) .
$$




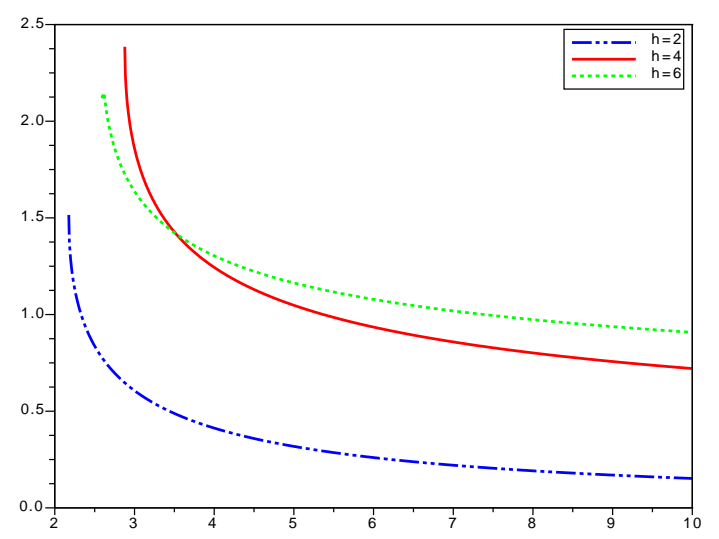

Fig. 18 Behavior of $\kappa$ (see (3.5)) with respect to $\alpha$ for three different values of the Hill exponent : $h=2, h=4$ and $h=6$.

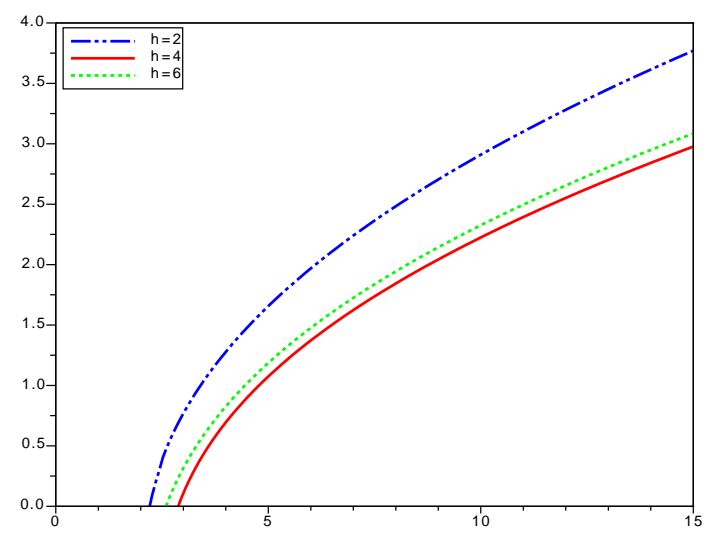

Fig. 19 Behavior of asymptotic velocity with respect to $\alpha$ for three different values of the Hill exponent : $h=2, h=4$ and $h=6$.

We plot in Figure 20 the quantity

$$
t=\frac{\Delta}{c^{*}}
$$

which is expected to be proportional to the switch time. At least it is a function of $h$, characteristic of the state transition. Again we note that this quantity is not monotone with respect to $h$ the Hill exponent. Nevertheless, for $\alpha$ small enough, the switch time is smaller for $h=4$ than for $h=2$.

\section{Conclusion and perspectives}

In this work, we proposed a simplified model for signal propagation in the MAPK cascade. It reduces the problem to a reaction-diffusion equation for the kinase MAP3K, the molecule Mos in the case of Xenopus oocytes. The propation of the downstream molecules of the cascade, MAP2K and MAPK, is slaved to the diffusion of Mos.

While certainly a strong simplification of the dynamics of the cascade, our model has allowed us to study a number of questions by analytic means and numerical approaches. While the model is too simplified to 


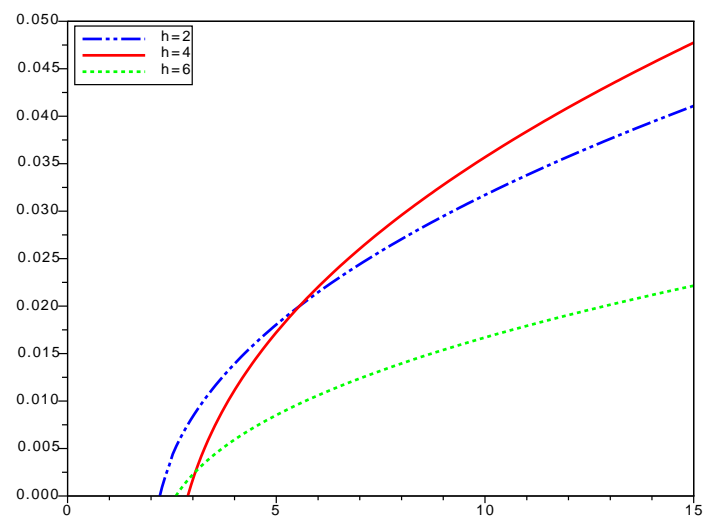

Fig. 20 Behavior of the switch time (see (5.7)) with respect to $\alpha$ for three different values of the Hill exponent : $h=2, h=4$ and $h=6$.

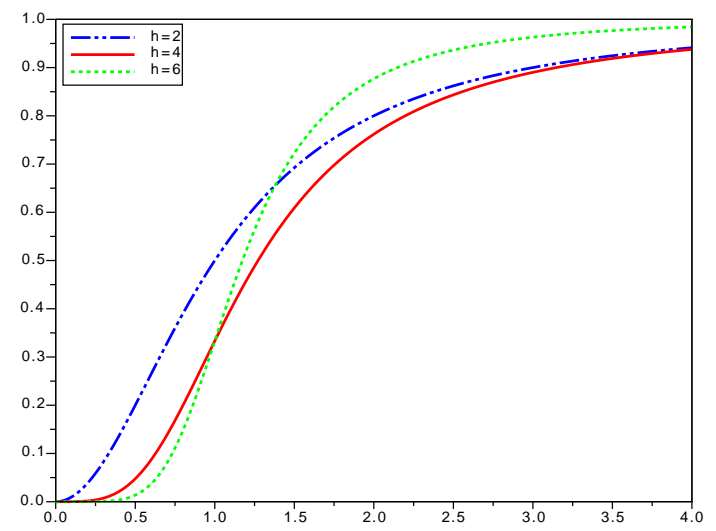

Fig. 21 Function $m \mapsto z_{\alpha}(m)$ for three different values of the Hill exponent $: h=2, h=4$ and $h=6$.

allow a direct comparison with experiment, its study has provided us with a number of key insights that merit further investigation in more elaborate models. Two results deserve special mention in this context. First, the role of the nonlinearity in the reaction term, hence the ultrasensitive response. The propagation speed depends on this nonlinearity, but in an non-intuitive way. There is no direct correlation between the Hill exponent $h$ and the asymptotic speed of propagation. Definitely the speed of propagation is not monotone with respect to $h$ (and the propagation speed is less for $h=4$ than for $h=2$ ). We do not wish to speculate too far about the possible biological consequences this fact might have, in any case it can be taken as an indication that optimization of the speed of the propagating front is not a design criterion for the signalling chain.

Second, and in our view even more intruiging, is the role played by the boundary and initial conditions. We have found that the propagation depends quite sensitively on the details of these conditions. In particular, deciding whether or not the signal propagates cannot be embodied in a single parameter (say the $L^{\infty}$ or the $L^{1}$ norm of the signal).

In our view, this points to an important feature that has so far not been present in the discussion of the functioning of the MAPK cascade. These discussions have largely been focused around the bistability - indeed, a precondition for all our discussion - and ultrasensitivity. Our result gives a hint at another dimension of the signaling process: how the molecular concentration at signal input is distributed has an effect on signal 
propagation. Molecular architectures inside the cell will therefore have to buffer concentration variations in order to control signal propagation. How the scenario which is present in our one-dimensional model will be modified in more realistic cellular geometries is, of course, an interesting open question.

\section{A Derivation of the expression of the reaction term $\Sigma[m(x, t)]$.}

The expression of the reaction term depends on the kinetics chosen for the signaling cascade. Typically, the cascade has two phosphorylation states so that for a two-level cascade with molecules $y_{0}$ and $z_{0}$, two respective phosphorylated states exist, for $y_{0}$ these are called $y_{1}$ and $y_{2}$, and for $z_{0}$, respectively, $z_{1}$ and $z_{2}$. Recall that $m$ denote the concentration of the molecule at the cascade entry. Two particular types of kinetics are linear and saturated, Michaelis-Menten kinetics. In the latter case, the equations read as, following [1] :

$$
\begin{aligned}
& \dot{y}_{0}=\frac{V_{6} y_{1}}{K_{6}+y_{1}}-\frac{V_{3} m y_{0}}{K_{3}+y_{0}} \\
& \dot{y}_{1}=-\left(\dot{y}_{0}+\dot{y}_{2}\right) \\
& \dot{y}_{2}=\frac{V_{4} m y_{1}}{K_{4}+y_{1}}-\frac{V_{5} y_{2}}{K_{5}+y_{2}} \\
& \dot{z}_{0}=\frac{V_{10} z_{1}}{K_{10}+z_{1}}-\frac{V_{7} y_{2} z_{0}}{K_{7}+z_{0}} \\
& \dot{z}_{1}=-\left(\dot{z}_{0}+\dot{z}_{2}\right) \\
& \dot{z}_{2}=\frac{V_{8} y_{2} z_{1}}{K_{8}+z_{1}}-\frac{V_{9} z_{2}}{K_{9}+z_{2}}
\end{aligned}
$$

where the $V_{i}$ and $K_{i}$ are reaction speeds and equilibrium constants, respectively, and where the numbering of the reactions follows the scheme by [5] which is also used in ref. [1]. This scheme simply numbers the reactions sequentially layer by layer, first all phosphorylations, and then all dephosphorylations. Note that if equilibrium constants are much larger than the molecular concentrations, the linear regime is recovered.

As shown in [10], for a particular symmetric choice of the parameters, the system can be rendered non-dimensional. For this parameter-symmetric case, the introduction of the functions

$$
w_{i} \equiv \frac{y_{i}}{1+y_{i}}, \quad q_{i} \equiv \frac{z_{i}}{1+z_{i}} .
$$

allows to rewrite the rhs of the equations in a simple polynomial form, from which the fixed-point value conditions are easy to read off. For the variable $w_{i}$, they are given by $w_{2}=m w_{1}=m^{2} w_{0}$.

Exploiting the conservation of molecules $y_{i}$ as expressed by the condition $\sum_{i=0,1,2} y_{i}=y_{T}$, one finds after suitable normalization the condition

$$
\frac{w_{1}}{1-w_{1}}+\frac{w_{2}}{1-w_{2}}+\frac{w_{3}}{1-w_{3}}=1
$$

which at the fixed-point reduces to a cubic equation, e.g. for $w_{0}$,

$$
4 m^{3} w_{0}^{3}-3 m\left(1+m+m^{2}\right) w_{0}^{2}+2\left(1+m+m^{2}\right) w_{0}-1=0 .
$$

which can be solved exactly. The case of linear kinetics can, however, also be recovered by ignoring the highest order terms of this equation. One then finds

$$
w_{0}=\frac{1}{2} \frac{1}{1+m+m^{2}}
$$

Although the full final expression for $w_{0}$ from the cubic equation does have a more involved form, the degree of the polynomial remains two as for the linear kinetics. From the fixed-point relation, we have

$$
w_{2}=\frac{1}{2} \frac{m^{2}}{1+m+m^{2}} .
$$


The same calculation can be repeated for the variable $q_{i}$. One obtains an equation of the same form as (A.9) in the variable $q_{1}$, only with $m$ replaced by

$$
s=v \frac{w_{2}}{1-w_{2}}
$$

where $v \equiv V_{7} / V_{10}$. The same argument can then be repeated leading to

$$
q_{2}=\frac{1}{2} \frac{s^{2}}{1+s+s^{2}}
$$

Putting the equations (A.11) and (A.13) together then leads already for the approximate linear kinetics to an involved expression, however with a polynomial of maximal degree four in the variable $m$ in both denominator and numerator. This remains the case also after the required transforms back from $w_{i}$ and $q_{i}$ to the original variables $y_{i}$ and $z_{i}$. While the full expression obtained from the two cubic equations can still be written down analytically, it turns out to be yet more involved and less illuminating. For both types of kinetics the Hill exponent is given by the product of the number of phosphorylation levels times cascade levels, hence four. For convenience in our mathematical treatment, we replace both involved expressions by the simple expression for $\Sigma[m(x, t)]$ used in the text since we are interested chiefly in the role of ultrasensitivity, i.e. on the value of the Hill coefficient, on wave propagation. In order to formulate the feedback loop in which MAPK acts back on Mos, $m$, we follow Angeli et al. [1] and write

$$
\dot{m}=-\gamma m+z_{2}
$$

in which $z_{2}=\Sigma[m]$ acts as the source term.

\section{B Properties of $q_{\beta}$ defined at (3.14)}

Let us recall the definitions. For any $\beta \in(\kappa, b)$, where $\kappa$ is defined at (3.5), the length $\ell_{\beta}$ is defined as follows

$$
\ell_{\beta}=2 \int_{0}^{\beta} \frac{1}{\sqrt{2(F(\beta)-F(u))}} \mathrm{d} u,
$$

where $F$ is defined at (3.2). Let $q_{\beta}$ be the solution of

$$
q_{\beta}^{\prime \prime}+f\left(q_{\beta}\right)=0 \text { in } \mathbb{R}^{+},
$$

with

$$
q_{\beta}(0)=0
$$

and

$$
\frac{1}{2} q_{\beta}^{\prime 2}+F\left(q_{\beta}\right)=F(\beta)
$$

Let us now show that these conditions imply

$$
q_{\beta}>0, q_{\beta}(0)=q_{\beta}\left(\ell_{\beta}\right)=0 \text { and } q_{\beta}(x) \leq q_{\beta}\left(\frac{\ell_{\beta}}{2}\right)=\beta \text { on }\left(0, \ell_{\beta}\right)
$$

$q_{\beta}\left(\frac{\ell_{\beta}}{2}\right)=\beta$

1. First, we determine the zeros of the first derivative of $q_{\beta}, q_{\beta}^{\prime}$. Thanks to the first integral (B.1), if there exists $x_{0}$ such that $q_{\beta}^{\prime}\left(x_{0}\right)=0$ then necessarily $F\left(q_{\beta}(x)\right)=F(\beta)$. Since $F$ is a bijection from $[\kappa, b]$ to $[0, F(b)]$ and non positive on $[0, \kappa]$ (see Figure 22), this implies $q\left(x_{0}\right)=\beta$.

2. From the definition,

$$
\frac{\ell_{\beta}}{2}=\int_{0}^{\beta} \frac{1}{\sqrt{2\left(F\left(q_{\beta}\left(x_{0}\right)\right)-F(u)\right)}} \mathrm{d} u .
$$

Since $q_{\beta}^{\prime}>0$ if $q_{\beta} \in(0, \beta)$, we can set the change of variable $u=q_{\beta}(y)$. On $(0, \beta)$, we have $q^{\prime}(y)=\sqrt{2\left(F\left(q_{\beta}\left(x_{0}\right)\right)-F\left(q_{\beta}(y)\right)\right)}$, thus we obtain

Finally, this shows that

$$
\frac{\ell_{\beta}}{2}=\int_{0}^{x_{0}} \mathrm{~d} y=x_{0} .
$$

$$
q_{\beta}\left(\frac{\ell_{\beta}}{2}\right)=\beta .
$$




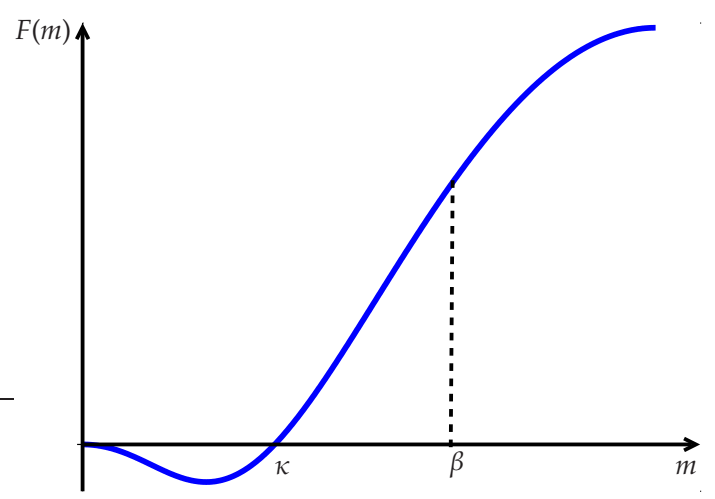

Fig. 22 Function $F(m)=\int_{0}^{x} f(u) \mathrm{d} u$.

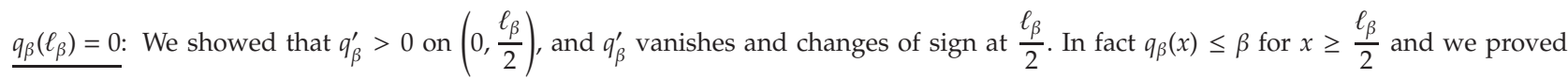
previously that $q^{\prime}$ vanishes in $x_{0}$ if and only if $q_{\beta}\left(x_{0}\right)=\beta$. Finally, $q_{\beta}^{\prime}<0$ on $\left(\frac{\ell_{\beta}}{2}, \beta\right)$. Let us define now $r(u)=q_{\beta}\left(\frac{\ell_{\beta}}{2}-u\right)$ and $s(u)=q_{\beta}\left(\frac{\ell_{\beta}}{2}+u\right)$. They are both solution of the following Cauchy problem:

$$
\begin{aligned}
y^{\prime \prime}+f(y) & =0 \\
y(0) & =\beta, \\
y^{\prime}(0) & =0 .
\end{aligned}
$$

As a consequence, $r(u)=s(u)$ on $\left[0, \frac{\ell_{\beta}}{2}\right]$. Thus,

$$
\begin{aligned}
q_{\beta}\left(\ell_{\beta}\right) & =\int_{0}^{\ell_{\beta}} q_{\beta}^{\prime}(t) \mathrm{d} t \\
& =\int_{0}^{\frac{\ell_{\beta}}{2}} q_{\beta}^{\prime}(t) \mathrm{d} t+\int_{\frac{\ell_{\beta}}{2}}^{\beta} q_{\beta}^{\prime}(t) \mathrm{d} t, \\
& =\int_{0}^{\frac{\ell_{\beta}}{2}} q_{\beta}^{\prime}\left(\frac{\ell_{\beta}}{2}+u\right) \mathrm{d} u+\int_{0}^{\frac{\ell_{\beta}}{2}} q_{\beta}^{\prime}\left(\frac{\ell_{\beta}}{2}+u\right) \mathrm{d} u \\
& =\int_{0}^{\frac{\ell_{\beta}}{2}} r^{\prime}(u) \mathrm{d} u-\int_{0}^{\frac{\ell_{\beta}}{2}} s^{\prime}(u) \mathrm{d} u \\
& =0 .
\end{aligned}
$$

\section{Acknowledgments}

The authors want to thank Frédéric Lagoutière for his fruitful remarks.

\section{References}

1. David Angeli, James E. Ferrell, and Eduardo D. Sontag. Detection of multistability, bifurcations, and hysteresis in a large class of biological positive-feedback systems. Proceedings of the National Academy of Sciences of the United States of America, 101(7):1822-1827, 2004. 
2. D. G. Aronson and H. F. Weinberger. Nonlinear diffusion in population genetics, combustion, and nerve propagation. Lecture Notes in Mathematics, 446:5-49, 1975.

3. A Goldbeter and D E Koshland. An amplified sensitivity arising from covalent modification in biological systems. Proceedings of the National Academy of Sciences of the United States of America, 78(11):6840-6844, 1981.

4. C. C. Govern and A. K. Chakraborty. Signaling cascades modulate the speed of signal propagation through space. PLoS ONE, 4(2):e4639, 2009.

5. B. N. Kholodenko. Negative feedback and ultrasensitivity can bring about oscillations in the mitogen-activated protein kinase cascades. Eur J Biochem, 267(6):1583-1588, Mar 2000.

6. Nick I. Markevich, Mikhail A. Tsyganov, Jan B. Hoek, and Boris N. Kholodenko. Long-range signaling by phosphoprotein waves arising from bistability in protein kinase cascade. Molecular Systems Biology, 2006. Article number : 61.

7. David O. Morgan. The Cell Cycle: Principles of Control. New Science Press, 2007.

8. J. D. Murray. Mathematical Biology, $2 d$ ed., volume 19 of Biomathematics. Springer, New York, 1993

9. Javier Muoz-Garca, Zoltan Neufeld, and Boris N. Kholodenko. Positional information generated by spatially distributed signaling cascades. PLoS Comput Biol, 5(3):e1000330, 032009.

10. C. Russo, C. Giuraniuc, R. Blossey, and J.-F. Bodart. On the equilibria of the mapk cascade: Cooperativity, modularity and bistability. Physica A: Statistical Mechanics and its Applications, 388(24):5070 - 5080, 2009. 